\title{
Relativistic quantum fluid with boost invariance
}

\author{
D. Rindori, ${ }^{1}$ L. Tinti ${ }^{2,3}$ F. Becattini ${ }^{1},{ }^{1}$ and D. H. Rischke ${ }^{2,4}$ \\ ${ }^{1}$ Università di Firenze and INFN Sezione di Firenze Via G. Sansone 1, Sesto Fiorentino, \\ I-50019 Florence, Italy \\ ${ }^{2}$ Institut für Theoretische Physik, Johann Wolfgang Goethe-Universität Max-von-Laue-Str. 1, \\ D-60438 Frankfurt am Main, Germany \\ ${ }^{3}$ Instytut Fizyki, Uniwersytet Jana Kochanowskiego w Kielcach ul. Uniwersytecka 7, \\ PL 25-406 Kielce, Poland \\ ${ }^{4}$ Helmholtz Research Academy Hesse for FAIR, Campus Riedberg, Max-von-Laue-Str. 12, \\ D-60438 Frankfurt am Main, Germany
}

(Received 9 March 2021; accepted 1 February 2022; published 7 March 2022)

\begin{abstract}
We study a relativistic fluid with longitudinal boost invariance in a quantum-statistical framework as an example of a solvable nonequilibrium problem. For the free quantum field, we calculate the exact form of the expectation values of the stress-energy tensor and the entropy current. For the stress-energy tensor, we find that a finite value can be obtained only by subtracting the vacuum of the density operator at some fixed proper time $\tau_{0}$. As a consequence, the stress-energy tensor acquires nontrivial quantum corrections to the classical free-streaming form.
\end{abstract}

DOI: 10.1103/PhysRevD.105.056003

\section{INTRODUCTION}

Spurred by a successful description of experimental data in high-energy nuclear collisions, relativistic hydrodynamics has recently made major progress, both regarding its theoretical foundations as well as its phenomenological applications. Lately, the quantum-statistical foundations of relativistic hydrodynamics have attracted a great deal of attention [1-7], in particular to describe quantum phenomena in relativistic fluids such as chirality [8] and polarization [9]. In a quantum-statistical framework, hydrodynamic quantities, such as the stress-energy tensor and conserved currents, are the expectation values of the corresponding quantum operators with respect to a suitable statistical (or density) operator $\widehat{\rho}$ :

$$
T^{\mu \nu}=\operatorname{tr}\left(\widehat{\rho} \widehat{T}^{\mu \nu}\right)_{\text {ren }},
$$

where the subscript "ren" implies renormalization of the otherwise divergent expectation value.

In general, the form of the stress-energy tensor and the currents crucially depends on the density operator. Exact expressions are known only in a few cases, including the familiar global thermodynamic equilibrium and, as a recent

Published by the American Physical Society under the terms of the Creative Commons Attribution 4.0 International license. Further distribution of this work must maintain attribution to the author(s) and the published article's title, journal citation, and DOI. Funded by SCOAP ${ }^{3}$. development, global thermodynamic equilibrium with rotation and acceleration. However, no exact form is known in local thermodynamic equilibrium, which is defined by $[1,2,10,11]$

$$
\widehat{\rho}_{\mathrm{LE}}=\frac{1}{Z} \exp \left[-\int_{\Sigma} \mathrm{d} \Sigma_{\mu}\left(\widehat{T}^{\mu \nu} \beta_{\nu}-{\widehat{\zeta j^{\mu}}}^{\mu}\right),\right.
$$

where $\beta(x)$ is a four-temperature field [equal to the fourvelocity $u(x)$ divided by the temperature $T(x)]$, and $\zeta(x)$ is a scalar field [equal to the ratio of the chemical potential $\mu(x)$ associated with the conserved current $\widehat{j}$ and the temperature]. The hypersurface $\Sigma$ is a three-dimensional spacelike hypersurface, on which the local equilibrium is defined. The calculation of expectation values of operators using Eq. (2) can be performed only in the hydrodynamic limit of slowly varying fields [1]. For the stress-energy tensor, the leading-order term coincides with the familiar perfect-fluid expression. Beyond this approximation, quantum corrections appear, which have been estimated by means of a perturbative expansion only in the globalequilibrium case [12].

Recently, Akkelin $[13,14]$ derived an exact solution of a particular nonequilibrium problem, a free neutral scalar field with the density operator:

$$
\widehat{\rho}=\frac{1}{Z} \exp \left[-\frac{1}{T(\tau)} \int_{\Sigma(\tau)} \mathrm{d} \Sigma_{\mu} \widehat{T}^{\mu \nu} u_{\nu}\right],
$$

with $\Sigma(\tau)$ being a proper-time $\tau$ hyperbola in the future light cone in two dimensions (see Fig. 1) and $u(x)$ the four- 


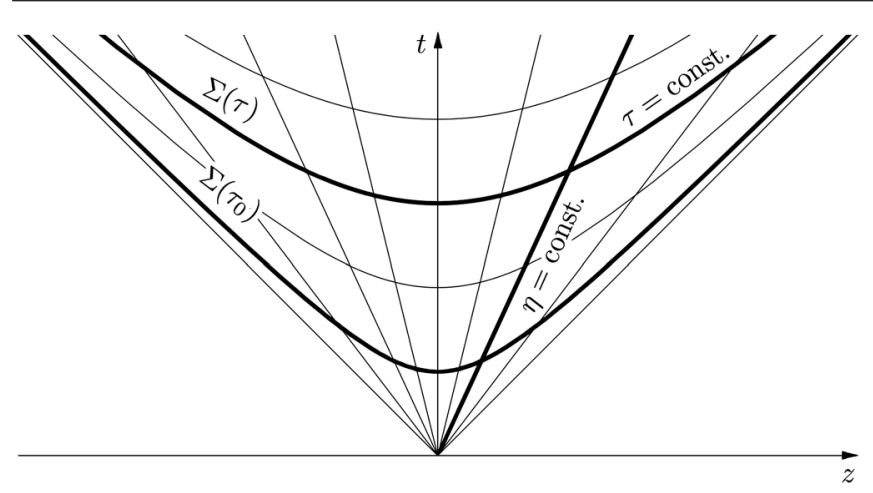

FIG. 1. Two-dimensional section of the future light cone. Curves of constant Milne time $\tau$ are hyperbolas, while curves of constant space-time rapidity $\eta$ are lines through the origin. The thicker hyperbolas are two-dimensional sections of the threedimensional hypersurfaces $\Sigma(\tau)$ and $\Sigma\left(\tau_{0}\right)$ at constant $\tau$ and constant $\tau_{0}$, respectively.

velocity field coinciding with the unit vector perpendicular to $\Sigma$. The density operator (3) is invariant under longitudinal boosts, a symmetry which has been often used to study general features of relativistic hydrodynamics problems. Lately, longitudinal boost-invariant solutions have been studied in the context of spin hydrodynamics [15] and magnetohydrodynamics [16-18]. This symmetry and the solution found in Ref. [13] also offers a special opportunity to explore in detail some essential features of quantum relativistic hydrodynamics in a nonequilibrium situation and, in particular, to determine the pure quantum corrections to classical hydrodynamics and kinetic equations, including those to the stress-energy tensor and to the entropy current. In other words, this solution provides a benchmark test of a relativistic quantum fluid.

In this work, we extend the results of Ref. [13] and study the stress-energy tensor with longitudinal boost-invariant symmetry. We find that, even for the simplest case of a free scalar field, there are relevant quantum corrections related to its renormalization by subtraction of the vacuum expectation value. Indeed, while the traditional vacuum of the field, expanded in plane waves, the so-called Minkowski vacuum, fails to provide a finite energy density, the subtraction of the vacuum expectation value with respect to the vacuum of the density operator does. Conversely, for the entropy current, no significant quantum correction is found.

This paper is organized as follows. We will start in Sec. II with a review of the density-operator approach in relativistic quantum-statistical mechanics with special emphasis on symmetry considerations. In Sec. III we will specialize to the symmetry of concern for this work, that is boost invariance. As underlying quantum field theory, in Sec. IV we will present the field theory of the free neutral scalar field in the future light cone, including a diagonalization of the density operator. This will put us in the position to calculate the thermal expectation value of the stress-energy tensor in Sec. V both in local thermodynamic equilibrium and out of equilibrium. Finally, we will discuss the entropy current and entropy production in Sec. VI, before concluding this paper in Sec. VII.

In this work, we use natural units $\hbar=c=k_{\mathrm{B}}=1$. Operators in Hilbert space are denoted with a wide upper hat, e.g., $\widehat{O}$, while vectors of unit length have a regular hat, that is $\hat{n}^{\mu}$. Repeated indices are assumed to be contracted. We adopt the "mostly minus" convention, so the Minkowski metric is $g_{\mu \nu}=\operatorname{diag}(1,-1,-1,-1)$. For the Levi-Civita symbol we use the convention $\epsilon^{0123}=1$.

\section{LOCAL THERMODYNAMIC EQUILIBRIUM, DENSITY OPERATOR, AND SYMMETRIES}

In quantum-statistical mechanics, the local-equilibrium density operator (LEDO) $\widehat{\rho}_{\text {LE }}$, Eq. (2), is obtained by maximizing the entropy $S=-\operatorname{Tr}\left(\widehat{\rho}_{\mathrm{LE}} \log \widehat{\rho}_{\mathrm{LE}}\right)$ under the constraints of fixed energy-momentum and, possibly, charge densities on a given three-dimensional spacelike hypersurface $\Sigma$. The hypersurface can be either specified a priori or can be found in a self-consistent procedure by using the thermodynamic fields themselves [1].

The energy-momentum densities on a hypersurface $\Sigma$ are obtained by contracting the stress-energy tensor with its normal unit vector $n$, so that the constraints read:

$$
n_{\mu} \operatorname{Tr}\left(\widehat{\rho}_{\mathrm{LE}} \widehat{T}^{\mu \nu}\right)_{\text {ren }}=n_{\mu} T^{\mu \nu},
$$

and likewise for the conserved currents. The densities on the right-hand side of Eq. (4) are meant to be the actual ones, no matter how they are known or defined, and they are supposedly finite. It is crucial to specify that the expectation values on the left-hand side must be suitably renormalized because, in general, the expectation value of the operator $\widehat{T}^{\mu \nu}$ with a density operator such as in Eq. (2) is divergent. For instance, in free field theory, the renormalization procedure is most readily established by subtracting the vacuum expectation value, that is,

$$
\operatorname{Tr}\left(\widehat{\rho} \widehat{T}^{\mu \nu}\right)_{\text {ren }}=\operatorname{Tr}\left(\widehat{\rho} \widehat{T}^{\mu \nu}\right)-\left\langle 0\left|\widehat{T}^{\mu \nu}\right| 0\right\rangle,
$$

which is tantamount to normal-ordering of the creation and annihilation operators because the currents are quadratic in the fields. We will delve into the question of vacuum subtraction in Sec. III A.

With the constraints (4), the function to be maximized with respect to $\widehat{\rho}_{\mathrm{LE}}$ is

$$
\begin{aligned}
& -\operatorname{Tr}\left(\widehat{\rho}_{\mathrm{LE}} \log \widehat{\rho}_{\mathrm{LE}}\right)+\int_{\Sigma(\tau)} \mathrm{d} \Sigma_{\mu}\left[\left(\operatorname{tr}\left(\widehat{\rho}_{\mathrm{LE}} \widehat{T}^{\mu \nu}\right)_{\text {ren }}-T^{\mu \nu}\right) \beta_{\nu}\right. \\
& \left.-\zeta\left(\operatorname{tr}\left(\widehat{\rho}_{\mathrm{LE}} \widehat{\hat{j}}^{\mu}\right)_{\mathrm{ren}}-j^{\mu}\right)\right]
\end{aligned}
$$


where the thermodynamic fields $\beta$ and $\zeta$ are Lagrange multipliers introduced to enforce the constraints (4). The solution is Eq. (2) and it should be pointed out that it can be kept in that simple form without subtraction of the vacuum expectation value because the latter is not an operator and would appear in the partition function $Z$ as well (in order to make $\operatorname{Tr} \widehat{\rho}_{\mathrm{LE}}=1$ ), hence canceling out in the ratio. With the energy-momentum densities given by the right-hand side of Eq. (4), the thermodynamic fields $\beta$ and $\zeta$ are determined by solving them with $\widehat{\rho}_{\text {LE }}$ given by Eq. (2); there are five equations with five unknowns $\left(\beta^{\mu}\right.$ and $\zeta$ ), which in general can be solved.

Unless $\beta$ is a Killing field and $\zeta$ constant, which characterizes a state of global thermodynamic equilibrium, the operator (2) is not independent of the hypersurface, hence it cannot be the actual density operator in the Heisenberg representation. In fact, the true density operator is, for a system which supposedly achieves local thermodynamic equilibrium at some time $\tau_{0}$, the so-called nonequilibrium density operator (NEDO), which is just Eq. (2) at time $\tau_{0}$ :

$$
\widehat{\rho}=\frac{1}{Z} \exp \left[-\int_{\Sigma\left(\tau_{0}\right)} \mathrm{d} \Sigma_{\mu}\left(\widehat{T}^{\mu \nu} \beta_{\nu}-\widehat{\zeta j}^{\mu}\right)\right] .
$$

This can be recast by using Gauss's theorem as [19]

$$
\begin{aligned}
\widehat{\rho}= & \frac{1}{Z} \exp \left[-\int_{\Sigma\left(\tau_{0}\right)} \mathrm{d} \Sigma_{\mu}\left(\widehat{T}^{\mu \nu} \beta_{\nu}-\widehat{j}^{\mu} \zeta\right)\right] \\
= & \frac{1}{Z} \exp \left[-\int_{\Sigma(\tau)} \mathrm{d} \Sigma_{\mu}\left(\widehat{T}^{\mu \nu} \beta_{\nu}-\widehat{j}^{\mu} \zeta\right)\right. \\
& \left.+\int_{\Omega} \mathrm{d} \Omega\left(\widehat{T}^{\mu \nu} \nabla_{\mu} \beta_{\nu}-\widehat{j}^{\mu} \nabla_{\mu} \zeta\right)\right] .
\end{aligned}
$$

In the exponent on the right-hand side, the first term is just the operator of local equilibrium at time $\tau$, while the second term contains dissipative corrections [19].

Suppose now that the actual density operator, the NEDO, has some symmetry, meaning that it commutes with some unitary representation $\widehat{U}(g)$ in Hilbert space of a group or a subgroup $\mathrm{G}$ of transformations, to be specific of the proper orthochronous Poincaré group $\mathrm{IO}(1,3)_{+}^{\uparrow}$. We have

$$
\begin{aligned}
\widehat{U}(g) \widehat{\rho} \widehat{U}(g)^{-1} & =\frac{1}{Z} \exp \left[-\int_{\Sigma\left(\tau_{0}\right)} \mathrm{d} \Sigma_{\mu}(x)\left(\hat{U}(g) \widehat{T}^{\mu \nu}(x) \widehat{U}(g)^{-1} \beta_{\nu}(x)-\zeta(x) \widehat{U}(g) \widehat{j}^{\mu}(x) \widehat{U}(g)^{-1}\right)\right] \\
& =\frac{1}{Z} \exp \left[-\int_{\Sigma\left(\tau_{0}\right)} \mathrm{d} \Sigma_{\mu}(x)\left(D\left(g^{-1}\right)_{\rho}^{\mu} D\left(g^{-1}\right)_{\sigma}^{\nu} \widehat{T}^{\rho \sigma}(g(x)) \beta_{\nu}(x)-\zeta(x) D\left(g^{-1}\right)_{\rho}^{\mu} \widehat{j}^{\rho}(g(x))\right)\right] .
\end{aligned}
$$

Let us now set $y=g(x)$ and we obtain, remembering $\mathrm{d} \Sigma_{\mu}(x)=D(g)_{\mu}^{\nu} \mathrm{d} \Sigma_{\nu}(y)$,

$$
\widehat{U}(g) \widehat{\rho} \widehat{U}(g)^{-1}=\frac{1}{Z} \exp \left[-\int_{g\left(\Sigma\left(\tau_{0}\right)\right)} \mathrm{d} \Sigma_{\rho}(y)\left(\widehat{T}^{\rho \sigma}(y) D\left(g^{-1}\right)_{\sigma}^{\nu} \beta_{\nu}\left(g^{-1}(y)\right)-\zeta\left(g^{-1}(y)\right) \widehat{j}^{\rho}(y)\right)\right] .
$$

Thus, if the hypersurface is invariant under the transformation $g$ and if

$$
D\left(g^{-1}\right)_{\sigma}^{\nu} \beta_{\nu}\left(g^{-1}(y)\right)=\beta_{\sigma}(y), \quad \zeta\left(g^{-1}(y)\right)=\zeta(y),
$$

then the operator $\hat{\rho}$ is invariant under the transformation $\widehat{U}(g) \widehat{\rho} \widehat{U}(g)^{-1}$. Equations (9) specify the symmetry conditions on the transformations of the thermodynamic fields $\beta$ and $\zeta$. An invariance of $\hat{\rho}$ has straightforward consequences for the expectation values of operators. For instance, for the stress-energy tensor,

$$
\begin{aligned}
T^{\mu \nu}(x) & =\operatorname{Tr}\left[\widehat{\rho} \widehat{T}^{\mu \nu}(x)\right]=\operatorname{Tr}\left[\widehat{\rho} \widehat{U}(g)^{-1} \widehat{T}^{\mu \nu}(x) \widehat{U}(g)\right] \\
& =D(g)_{\rho}^{\mu} D(g)_{\sigma}^{\nu} \operatorname{Tr}\left[\widehat{\rho} \widehat{T}^{\mu \nu}\left(g^{-1}(x)\right)\right] \\
& =D(g)_{\rho}^{\mu} D(g)_{\sigma}^{\nu} T^{\mu \nu}\left(g^{-1}(x)\right) .
\end{aligned}
$$

If we consider a one-parameter subgroup of transformations $g_{\phi}$ [e.g., a rotation, $g_{\phi}=\exp (-i \phi \mathbf{J})$, around some axis], Eqs. (9) and (10) have the consequence that the Lie derivative along the vector field $X(x)=\mathrm{d} g_{\phi}(x) / \mathrm{d} \phi$ of the field under consideration vanishes, that is,

$$
\mathcal{L}_{X}(\beta)^{\mu}=0, \quad \mathcal{L}_{X}(T)^{\mu \nu}=0 .
$$

An important question concerns the persistence of the symmetry of the local thermodynamic equilibrium operator, that is whether the implication

$$
\widehat{\rho}=\widehat{U}(g) \widehat{\rho} \widehat{U}(g)^{-1} \Rightarrow \widehat{\rho}_{\mathrm{LE}}(\tau)=\widehat{U}(g) \widehat{\rho}_{\mathrm{LE}}(\tau) \widehat{U}(g)^{-1}
$$

is true for any $\tau$. Indeed, it can be shown that if the subgroup $\mathrm{G}$ transforms $\Sigma\left(\tau_{0}\right)$ into itself and if the fields $\beta$ and $\zeta$ are also symmetric under $\mathrm{G}$, namely they fulfill Eqs. (9) or 
(11), this is the case. Indeed, by definition, $\widehat{\rho}_{\mathrm{LE}}(\tau)$ is the solution of maximizing a function which is invariant under any unitary transformation, the entropy, with the constraint (4). If a particular $\widehat{\rho}_{\text {LE }}$ fulfills Eq. (4), so will $\widehat{U}(g) \widehat{\rho}_{\mathrm{LE}}(\tau) \widehat{U}(g)^{-1}$ as it can be readily checked. Therefore, either $\widehat{U}(g) \widehat{\rho}_{\mathrm{LE}}(\tau) \widehat{U}(g)^{-1}$ is a different solution of the constrained maximization problem, or it coincides with $\widehat{\rho}_{\text {LE }}(\tau)$. In both cases, it is possible to generate one symmetric solution under the subgroup $G$ by using a particular solution $\widehat{\rho}_{\mathrm{LE}}^{(0)}$ and summing over all $g$ 's:

$$
\widehat{\rho}_{\mathrm{LE}}(\tau)=\frac{1}{M(G)} \sum_{g \in G} \widehat{U}(g) \widehat{\rho}_{\mathrm{LE}}^{(0)} \widehat{U}(g)^{-1} .
$$

It is then obvious that the sufficient condition for $\widehat{\rho}_{\mathrm{LE}}(\tau)$, given by Eq. (2), to be symmetric under $\mathrm{G}$ is that the fields $\beta$ and $\zeta$ fulfill Eqs. (9) at time $\tau$. This is a crucial point for the purpose of this work.

\section{RELATIVISTIC QUANTUM FLUID WITH LONGITUDINAL BOOST INVARIANCE}

Suppose that the density operator is given by Eq. (7) with $\Sigma\left(\tau_{0}\right)$ being the hyperboloid $\tau=\sqrt{t^{2}-z^{2}}=\tau_{0} \quad$ in Minkowski space-time and with

$$
\beta^{\mu}=\frac{1}{T\left(\tau_{0}\right)} \frac{1}{\tau_{0}}(t, 0,0, z)=\frac{1}{T\left(\tau_{0}\right)} u^{\mu},
$$

where $T\left(\tau_{0}\right)$ and $\zeta\left(\tau_{0}\right)$ are constant on the hypersurface. This vector field is timelike on the hypersurface $\Sigma\left(\tau_{0}\right)$, hence thermodynamically meaningful.

The field $\beta$ in Eq. (12) and the field $\zeta$ fulfill Eq. (9) for any longitudinal boost with hyperbolic angle $\xi$ along the $z$ axis, $L_{z}(\xi)$, and manifestly for translations and rotations in the $x y$ plane. Besides, the hypersurface $\Sigma\left(\tau_{0}\right)$ is invariant under the same transformations. Therefore, the density operator has the symmetry group $\mathrm{ISO}(2) \otimes \mathrm{SO}(1,1)$, that is the Euclidean group in the transverse plane times Lorentz transformations in the longitudinal direction. Furthermore, the density operator is also invariant under a spacereflection transformation turning $x, y, z$ into $-x,-y,-z$.

This symmetry group dictates the possible forms of vector and tensor fields, which are most easily found by using Milne coordinates, $(\tau, x, y, \eta)$, instead of the usual Cartesian ones, $(t, x, y, z)$ :

$$
\begin{aligned}
t & =\tau \cosh \eta, \quad z=\tau \sinh \eta, \\
\tau & =\sqrt{t^{2}-z^{2},} \quad \eta=\frac{1}{2} \log \left(\frac{t+z}{t-z}\right),
\end{aligned}
$$

such that the coordinate basis vectors are

$$
\begin{aligned}
\frac{\partial}{\partial \tau} & =\frac{1}{\tau}(t, 0,0, z)=(\cosh \eta, 0,0, \sinh \eta)=u, \\
\frac{\partial}{\partial \eta} & =\tau(\sinh \eta, 0,0, \cosh \eta)=(z, 0,0, t) \equiv \tau \widehat{\eta}, \\
\frac{\partial}{\partial x} & =\widehat{i}, \quad \frac{\partial}{\partial y}=\widehat{j},
\end{aligned}
$$

and the metric tensor is

$$
\mathrm{d} s^{2}=\mathrm{d} t^{2}-\mathrm{d} x^{2}-\mathrm{d} y^{2}-\mathrm{d} z^{2}=\mathrm{d} \tau^{2}-\mathrm{d} x^{2}-\mathrm{d} y^{2}-\tau^{2} \mathrm{~d} \eta^{2} .
$$

The vector fields $X(x)$ associated with the symmetry group along which the Lie derivatives vanish can be readily found:

$$
\begin{aligned}
& \frac{\mathrm{dT}_{1}(a) x}{\mathrm{~d} a}=\hat{i}, \quad \frac{\mathrm{dT}_{2}(a) y}{\mathrm{~d} a}=\hat{j}, \\
& \frac{\mathrm{dR}(\phi) x}{\mathrm{~d} \phi}=(0,-y, x, 0) \equiv r \hat{\varphi}, \quad \frac{\mathrm{dL}_{3}(\xi) x}{\mathrm{~d} \xi}=(z, 0,0, t)=\tau \hat{\eta},
\end{aligned}
$$

where $\mathrm{T}_{i}$ are translations in the coordinate directions of the $x y$ plane, $r=\sqrt{x^{2}+y^{2}}, \mathrm{R}(\varphi)$ is a rotation with angle $\varphi$ in the same plane, and $L_{3}(\xi)$ is a longitudinal boost with hyperbolic angle $\xi$. Note that three vector fields are just the Milne-coordinate basis vectors, which, by construction, have vanishing Lie derivatives among each other, that is vanishing Lie commutators.

As has been mentioned, the condition of vanishing Lie derivatives along the vector fields (13) puts strong limitations on the form of the fields in general. For instance, a vector field $V(x)$ can be decomposed onto the coordinate basis vectors:

$$
V(x)=A(\tau) u+B(\tau) \widehat{i}+C(\tau) \widehat{j}+D(\tau) \tau \widehat{\eta},
$$

where the coefficients depend on the variable $\tau$ only as a consequence of $\mathcal{L}_{X}(V)=0$, where $X$ is either $\widehat{i}$, or $\widehat{j}$, or $\tau \widehat{\eta}$. Also, by implementing $\mathcal{L}_{\hat{\varphi}}(V)=0$ one obtains that both $B$ and $C$ are in fact zero. Furthermore, by reflection invariance, the component proportional to $\hat{\eta}$ must be vanishing because a reflection turns $\eta$ into $-\eta$ and the vector field has just one component:

$$
V(x)=A(\tau) u .
$$

Similarly, the form a symmetric tensor field like the stress-energy tensor $T^{\mu \nu}$ can be obtained by iterated projections onto vectors and orthogonal components. The result is

$T^{\mu \nu}=\mathcal{E}(\tau) u^{\mu} u^{\nu}+\mathcal{P}_{\mathrm{T}}(\tau)\left(\hat{i}^{\mu} \hat{i}^{\nu}+\hat{j}^{\mu} \hat{j}^{\nu}\right)+\mathcal{P}_{\mathrm{L}}(\tau) \hat{\eta}^{\mu} \hat{\eta}^{\nu}$. 
The form (15) is different from the usual perfect fluid form, for which $\mathcal{P}_{\mathrm{T}}=\mathcal{P}_{\mathrm{L}}$. The difference between transverse and longitudinal pressure is due to the lack of full rotational symmetry.

In order to determine the three scalar functions in Eq. (15), we have to calculate the expectation values of operators with the density operator (7). The unit four-vector orthogonal to the hyperboloid with fixed $\tau$ is $u$ itself, so the operator (7) becomes

$$
\widehat{\rho}=\frac{1}{Z} \exp \left[-\frac{\widehat{\Pi}\left(\tau_{0}\right)}{T\left(\tau_{0}\right)}\right],
$$

with

$$
\widehat{\Pi}\left(\tau_{0}\right)=\int_{\Sigma\left(\tau_{0}\right)} \mathrm{d} \Sigma u_{\mu} u_{\nu} \widehat{T}^{\mu \nu}=\tau_{0} \int \mathrm{d} x \mathrm{~d} y \mathrm{~d} \eta u_{\mu} u_{\nu} \widehat{T}^{\mu \nu},
$$

where we have used the measure in Milne coordinates. It should be stressed that the operator $\widehat{\Pi}\left(\tau_{0}\right)$ is not conserved because the divergence of the integrand is not zero:

$$
\partial_{\mu}\left(u_{\nu} \widehat{T}^{\mu \nu}\right)=\widehat{T}^{\mu \nu} \partial_{\mu} u_{\nu} \neq 0,
$$

so it depends on $\tau_{0}$. We can also write down a general form of the local equilibrium operator $\widehat{\rho}_{\mathrm{LE}}(\tau)$ at any Milne time $\tau$ by taking the hyperboloid $\tau=$ const. as local-equilibrium hypersurface, which is invariant under the same transformations as $\Sigma\left(\tau_{0}\right)$, according to the discussion in Sec. II. Since the field $\beta(\tau)$ must fulfill Eqs. (9) and (11), it can only be of the form (14):

$$
\beta=\frac{1}{T(\tau)} u=\frac{1}{T(\tau)}(\cosh \eta, 0,0, \sinh \eta),
$$

thus the constraint (4) becomes, by using Eq. (15),

$$
\begin{aligned}
n_{\mu} \operatorname{Tr}\left(\widehat{\rho}_{\mathrm{LE}} \widehat{T}^{\mu \nu}\right)_{\mathrm{ren}} & \equiv n_{\mu} T_{\mathrm{LE}}^{\mu \nu}=u_{\mu} T_{\mathrm{LE}}^{\mu \nu} \\
& =\mathcal{E}(\tau)_{\mathrm{LE}} u^{\nu}=n_{\mu} T^{\mu \nu}=\mathcal{E}(\tau) u^{\nu}
\end{aligned}
$$

This vector equation comes down to one scalar equation $\mathcal{E}(\tau)_{\mathrm{LE}}=\mathcal{E}(\tau)$ with $T(\tau)$ as unknown to be determined once the actual $\mathcal{E}(\tau)$ is determined by using the actual density operator (7). The local thermodynamic equilibrium operator will be of the same form as Eq. (16), that is,

$$
\widehat{\rho}_{\mathrm{LE}}(\tau)=\frac{1}{Z} \exp \left[-\frac{\widehat{\Pi}(\tau)}{T(\tau)}\right],
$$

with $\widehat{\Pi}(\tau) \neq \widehat{\Pi}\left(\tau_{0}\right)$.

\section{A. Vacuum effects}

A very interesting feature of a relativistic quantum fluid with the four-temperature field (12) is that the spectrum of
$\widehat{\Pi}\left(\tau_{0}\right)$, and particularly the lowest-lying eigenvector, the $\widehat{\Pi}$ vacuum, may depend on $\tau$, as it is clear from Ref. [13]. This $\tau$-dependent vacuum $\left|0_{\tau}\right\rangle$ is in general also different from the vacuum of a quantum field theory-even for free fields - in flat space-time obtained by quantizing in Cartesian coordinates, the so-called Minkowski vacuum $\left|0_{M}\right\rangle$. This is clearly at variance with familiar equilibrium quantum thermodynamics, where the Hamiltonian operator achieves its minimal eigenvalue in the Minkowski vacuum. The distinction between vacua is very important as far as the renormalization of several quantities is concerned, including, e.g., the stress-energy tensor. In a free field theory, the renormalization of the expectation value of an operator $\widehat{O}$ involves the subtraction of its vacuum expectation value. If more vacua are present, there is an ambiguity as we could define, as usual,

$$
\langle\widehat{O}\rangle_{\text {ren }} \equiv \operatorname{Tr}(\widehat{\rho} \widehat{O})-\left\langle 0_{M}|\widehat{O}| 0_{M}\right\rangle,
$$

[see Eq. (5)] or, in our case,

$$
\langle\widehat{O}\rangle_{\text {ren }} \equiv \operatorname{Tr}(\widehat{\rho} \widehat{O})-\left\langle 0_{\tau}|\widehat{O}| 0_{\tau}\right\rangle .
$$

Note that the $\widehat{\Pi}$ vacuum can be subtracted by taking the limit $T(\tau) \rightarrow 0$ of the unrenormalized expression since

$$
\lim _{T(\tau) \rightarrow 0} \widehat{\rho}_{\mathrm{LE}}(\tau)=\lim _{T(\tau) \rightarrow 0} \frac{1}{\operatorname{Z}} \exp [-\widehat{\Pi}(\tau) / T(\tau)]=\left|0_{\tau}\right\rangle\left\langle 0_{\tau}\right| \equiv \mathrm{P}_{0_{\tau}} .
$$

For this reason, in general the vacuum $\left|0_{\tau}\right\rangle$ will have the same symmetries as the original density operator, but it will be less symmetric than the supposedly Poincaré-invariant Minkowski vacuum $\left|0_{M}\right\rangle{ }^{1}$

It should be pointed out that the vacuum $\left|0_{\tau}\right\rangle$ is $\tau$ dependent, hence a subtraction like in Eq. (20) implies that the expectation value can get an undesired time dependence. For instance, if we define the renormalized stress-energy tensor as

$$
T^{\mu \nu} \equiv \operatorname{Tr}\left(\widehat{\rho} \widehat{T}^{\mu \nu}\right)-\left\langle 0_{\tau}\left|\widehat{T}^{\mu \nu}\right| 0_{\tau}\right\rangle=\operatorname{Tr}\left[\left(\widehat{\rho}-\mathrm{P}_{0_{\tau}}\right) \widehat{T}^{\mu \nu}\right],
$$

then

$$
\begin{aligned}
\partial_{\mu} T^{\mu \nu} & =\partial_{\mu} \operatorname{Tr}\left[\left(\widehat{\rho}-\mathrm{P}_{0_{\tau}}\right) \widehat{T}^{\mu \nu}\right] \\
& =\operatorname{Tr}\left[\left(\widehat{\rho}-\mathrm{P}_{0_{\tau}}\right) \partial_{\mu} \widehat{T}^{\mu \nu}\right]+\operatorname{Tr}\left[-\left(\partial_{\mu} \mathrm{P}_{0_{\tau}}\right) \widehat{T}^{\mu \nu}\right] \\
& =-\operatorname{Tr}\left[u_{\mu} \frac{\partial \mathrm{P}_{0_{\tau}}}{\partial \tau} \widehat{T}^{\mu \nu}\right] \neq 0,
\end{aligned}
$$

where we used $\partial_{\mu} \widehat{T}^{\mu \nu}=0$ and the time independence of the density operator. Therefore, the expectation value $T^{\mu \nu}$

\footnotetext{
${ }^{1}$ This does not mean that the vacuum $\left|0_{\tau}\right\rangle$ is degenerate, but that Poincaré transformations will give rise to nonvanishing components of excited states.
} 
would no longer fulfill a conservation equation even though the operator $\widehat{T}^{\mu \nu}$ does.

Therefore, in order to have a properly finite, conserved stress-energy tensor for a relativistic quantum fluid, the vacuum must be necessarily fixed, just like the density operator. Of course the Minkowski vacuum $\left|0_{M}\right\rangle$ meets this requirement and is seemingly the most obvious choice. However, we will see in Sec. V that the subtraction of the vacuum expectation value of $\widehat{T}^{\mu \nu}$ of a free field with respect to $\left|0_{M}\right\rangle$ does not give rise to a finite value, for the particular symmetry we are dealing with, and an alternative definition is needed.

\section{FREE SCALAR FIELD IN MILNE COORDINATES}

As has been mentioned in the Introduction, a closed analytic form of the stress-energy tensor with the fourtemperature field (12) exists for the case of free fields, providing the opportunity to determine exact quantum corrections to the classical expressions in the nonequilibrium case. The system which is described by the operator (7) and a free scalar field is that of a fluid where interactions effectively cease at the hypersurface $\Sigma\left(\tau_{0}\right)$ with temperature $T\left(\tau_{0}\right)$ and a four-velocity $u=T \beta$, with particles freely streaming thereafter. We thus expect to recover, in the classical limit, the classical kinetictheory solutions of the free-streaming Boltzmann equation starting from the local thermodynamic equilibrium expressions with proper temperature $T\left(\tau_{0}\right)$ and flow velocity $u\left(\tau_{0}\right)$.

The calculation of the stress-energy tensor for the massive free scalar field $\widehat{\psi}(x)$ requires the solution of the Klein-Gordon equation in Milne coordinates:

$$
\left[\frac{1}{\tau} \partial_{\tau}\left(\tau \partial_{\tau}\right)-\partial_{x}^{2}-\partial_{y}^{2}-\frac{1}{\tau^{2}} \partial_{\eta}^{2}+m^{2}\right] \widehat{\psi}\left(\tau, \mathbf{x}_{\mathrm{T}}, \eta\right)=0 .
$$

This is a well-known problem in the literature [20,21], which has even raised some discussion. It has been convincingly demonstrated [21] that, within the future light cone, there is a complete set of solutions of the Klein-Gordon equation in Milne coordinates, which allow an expansion in terms of the familiar plane waves and which do not mix positive and negative frequencies. These mode functions can be obtained starting from the usual expansion of the scalar field [13] in plane waves. We will recapitulate the salient points of the derivation presented in Ref. [13]. The obtained full expansion of the field in Milne coordinates reads

$$
\begin{aligned}
\widehat{\psi}\left(\tau, \mathbf{x}_{\mathrm{T}}, \eta\right)= & \int \frac{\mathrm{d}^{2} p_{T} \mathrm{~d} \mu}{4 \pi \sqrt{2}}\left[h(\mathbf{p}, \tau) \mathrm{e}^{i\left(\mathbf{p}_{\mathrm{T}} \cdot \mathbf{x}_{\mathrm{T}}+\mu \eta\right)} \hat{b}_{\mathrm{p}}\right. \\
& \left.+h^{*}(\mathbf{p}, \tau) \mathrm{e}^{-i\left(\mathbf{p}_{\mathrm{T}} \cdot \mathbf{x}_{\mathrm{T}}+\mu \eta\right)} \widehat{b}_{\mathrm{p}}^{\dagger}\right]
\end{aligned}
$$

where $\mathbf{p}=\left(\mathbf{p}_{\mathrm{T}}, \mu\right)$ to distinguish it from the Cartesian vector $\mathbf{p}=\left(\mathbf{p}_{\mathrm{T}}, p_{z}\right)$. Here, $\widehat{b}_{\mathrm{p}}^{\dagger}$ and $\widehat{b}_{\mathrm{p}}$ are creation and annihilation operators satisfying the usual algebra:

$\left[\widehat{b}_{\mathrm{p}}, \widehat{b}_{\mathrm{p}^{\prime}}^{\dagger}\right]=\delta^{2}\left(\mathbf{p}_{\mathrm{T}}-\mathbf{p}_{\mathrm{T}}^{\prime}\right) \delta\left(\mu-\mu^{\prime}\right), \quad\left[\widehat{b}_{\mathrm{p}}, \widehat{b}_{\mathrm{p}^{\prime}}\right]=0=\left[\widehat{b}_{\mathrm{p}}^{\dagger}, \widehat{b}_{\mathrm{p}^{\prime}}^{\dagger}\right]$.

The relation between the operators $\widehat{b}_{\mathrm{p}}^{\dagger}$ and the familiar $\widehat{a}^{\dagger}(p)$ of the plane-wave expansion reads

$$
\widehat{a}^{\dagger}(p)=\frac{1}{\sqrt{2 \pi m_{\mathrm{T}} \cosh y}} \int_{-\infty}^{+\infty} \mathrm{d} \mu \mathrm{e}^{-i \mu y} \widehat{b}_{\mathrm{p}}^{\dagger},
$$

where $y$ is the particle rapidity in longitudinal direction, which can be easily inverted to obtain $p_{z}$. Since there is no mixing between creation and annihilation operators, the vacuum of the $b_{\mathrm{p}}$ operators is the same Minkowski vacuum $\left|0_{M}\right\rangle$ as for the operators $a(p)$, which is a consequence of the fact that the functions $h(\mathrm{p}, \tau)$ can be expressed as a linear combination of plane waves with just positive frequency [22]. In Eq. (21) $\mu$ is the eigenvalue of the boost operator $\widehat{K}_{z}$, so that

$$
\widehat{U}\left(\mathrm{~L}_{3}(\xi)\right) \widehat{b}_{\mathrm{p}}^{\dagger} \widehat{U}\left(\mathrm{~L}_{3}(\xi)\right)^{-1}=\mathrm{e}^{-i \xi \widehat{K}_{z}} \widehat{b}_{\mathrm{p}}^{\dagger} \mathrm{e}^{i \xi \widehat{K}_{z}}=\mathrm{e}^{-i \xi \mu} \widehat{b}_{\mathrm{p}}^{\dagger},
$$

i.e., $\widehat{b}_{\mathrm{p}}^{\dagger}$ creates a state with eigenvalue $\mu$. The $\tau$-dependent functions in Eq. (21) are

$h(\mathrm{p}, \tau)=-i \mathrm{e}^{2}{ }^{\frac{\pi}{\mu}} \mathrm{H}_{i \mu}^{(2)}\left(m_{\mathrm{T}} \tau\right), \quad h^{*}(\mathrm{p}, \tau)=i \mathrm{e}^{-\frac{\pi}{2} \mu} \mathrm{H}_{i \mu}^{(1)}\left(m_{\mathrm{T}} \tau\right)$,

where the Hankel functions are [23]

$$
\begin{aligned}
& \mathrm{H}_{i \mu}^{(2)}\left(m_{\mathrm{T}} \tau\right)=-\frac{1}{i \pi} \mathrm{e}^{-\frac{\pi}{2} \mu} \int_{-\infty}^{+\infty} \mathrm{d} \theta \mathrm{e}^{-i m_{\mathrm{T}} \tau \cosh \theta+i \mu \theta}, \\
& \mathrm{H}_{i \mu}^{(1)}\left(m_{\mathrm{T}} \tau\right)=\frac{1}{i \pi} \mathrm{e}^{\frac{\pi}{2} \mu} \int_{-\infty}^{+\infty} \mathrm{d} \theta \mathrm{e}^{i m_{\mathrm{T}} \tau \cosh \theta-i \mu \theta},
\end{aligned}
$$

with $m_{\mathrm{T}}=\sqrt{\mathrm{p}_{\mathrm{T}}^{2}+m^{2}}$ being the transverse mass. The integration variable $\theta$ in Eq. (25) is related to the Milne coordinates and rapidity by [13]

$$
\theta=y-\eta
$$

The functions (25) solve the differential equations:

$$
\left[\frac{1}{\tau} \partial_{\tau}\left(\tau \partial_{\tau}\right)+m_{T}^{2}+\frac{\mu^{2}}{\tau^{2}}\right] h(\mathrm{p}, \tau)=0,
$$

which are indeed Bessel's differential equations. It is also useful to define 


$$
\omega^{2}=m_{\mathrm{T}}^{2}+\frac{\mu^{2}}{\tau^{2}}
$$

Let us now work out the density operator, particularly the operator $\widehat{\Pi}(\tau)$ in Eq. (18). In a nonequilibrium situation it is known that the density operator depends on the particular stress-energy tensor operator which is employed; however, for the free scalar field we will be using the canonical tensor:

$$
\begin{aligned}
\widehat{T}_{C}^{\mu \nu} & =\frac{1}{2}\left(\partial^{\mu} \widehat{\psi} \partial^{\nu} \widehat{\psi}+\partial^{\nu} \widehat{\psi} \partial^{\mu} \widehat{\psi}\right)-g^{\mu \nu} \widehat{\mathcal{L}}, \\
\widehat{\mathcal{L}} & =\frac{1}{2}\left(g^{\mu \nu} \partial_{\mu} \widehat{\psi} \partial_{\nu} \widehat{\psi}-m^{2} \widehat{\psi}^{2}\right),
\end{aligned}
$$

where $\widehat{\mathcal{L}}$ is the Lagrangian density. Hence,

$$
\begin{aligned}
\widehat{T}_{C}^{\mu \nu} u_{\mu} u_{\nu}= & \frac{1}{2}\left[\left(\partial_{\tau} \widehat{\psi}\right)^{2}+\left(\partial_{x} \widehat{\psi}\right)^{2}\right. \\
& \left.+\left(\partial_{y} \widehat{\psi}\right)^{2}+\frac{1}{\tau^{2}}\left(\partial_{\eta} \widehat{\psi}\right)^{2}+m^{2} \widehat{\psi}^{2}\right] .
\end{aligned}
$$

By using the above equation along with Eq. (21) and taking advantage of the invariance by reflection $p \rightarrow-p$ of the functions $h(\mathrm{p}, \tau)$, one can obtain the following expression for $\widehat{\Pi}(\tau)$ :

$$
\begin{aligned}
\widehat{\Pi}(\tau)= & \tau \int \mathrm{d} x \mathrm{~d} y \mathrm{~d} \eta \widehat{T}^{\mu \nu} u_{\mu} u_{\nu} \\
= & \int \mathrm{d}^{2} p_{T} \mathrm{~d} \mu \frac{\omega}{2}\left[K\left(\widehat{b}_{\mathrm{p}} \widehat{b}_{\mathrm{p}}^{\dagger}+\widehat{b}_{\mathrm{p}}^{\dagger} \widehat{b}_{\mathrm{p}}\right)\right. \\
& \left.+\Lambda \widehat{b}_{\mathrm{p}} \widehat{b}_{-\mathrm{p}}+\Lambda^{*} \widehat{b}_{\mathrm{p}}^{\dagger} \widehat{b}_{-\mathrm{p}}^{\dagger}\right],
\end{aligned}
$$

where the positive real function $K(\mathrm{p}, \tau)$ and the complex function $\Lambda(p, \tau)$ are defined as

$$
\begin{aligned}
K(\mathrm{p}, \tau) & =\frac{\pi \tau}{4 \omega}\left(\left|\partial_{\tau} h(\mathrm{p}, \tau)\right|^{2}+\omega^{2}|h(\mathrm{p}, \tau)|^{2}\right), \\
\Lambda(\mathrm{p}, \tau) & =\frac{\pi \tau}{4 \omega}\left\{\left[\partial_{\tau} h(\mathrm{p}, \tau)\right]^{2}+\omega^{2} h^{2}(\mathrm{p}, \tau)\right\} .
\end{aligned}
$$

Note that, with $\omega$ and $h$ being invariant under a reflection $\mathrm{p} \rightarrow-\mathrm{p}$, so are $K$ and $\Lambda$, and

$$
K^{2}(\mathrm{p}, \tau)-|\Lambda(\mathrm{p}, \tau)|^{2}=1,
$$

as $K^{2}-|\Lambda|^{2}$ is proportional to the Wronskian of the Hankel functions

$$
\begin{aligned}
& K^{2}(\mathrm{p}, \tau)-|\Lambda(\mathrm{p}, \tau)|^{2} \\
& \quad=-\left(\frac{\pi m_{\mathrm{T}} \tau}{4}\right)^{2}\left(W\left[\mathrm{H}_{i \mu}^{(2)}\left(m_{\mathrm{T}} \tau\right), \mathrm{H}_{i \mu}^{(1)}\left(m_{\mathrm{T}} \tau\right)\right]\right)^{2},
\end{aligned}
$$

which is known to be a very simple function [23]:

$$
\begin{aligned}
W\left[\mathrm{H}_{i \nu}^{(2)}(x), \mathrm{H}_{i \nu}^{(1)}(x)\right] & =\mathrm{H}_{i \nu}^{(2)^{\prime}}(x) \mathrm{H}_{i \nu}^{(1)}(x)-\mathrm{H}_{i \nu}^{(1)^{\prime}}(x) \mathrm{H}_{i \nu}^{(2)}(x) \\
& =\frac{4 i}{\pi x}
\end{aligned}
$$

The above relation is not accidental but it is related to the invariance of the Klein-Gordon scalar product of the mode functions [22]. Equation (32) allows one to write

$$
\begin{aligned}
& K(\mathrm{p}, \tau)=\cosh 2 \Theta(\mathrm{p}, \tau) \\
& \Lambda(\mathrm{p}, \tau)=\sinh 2 \Theta(\mathrm{p}, \tau) \exp [i \chi(\mathrm{p}, \tau)],
\end{aligned}
$$

which is very important to highlight the vacuum effects, as it will become clear later.

Due to the terms proportional to $\Lambda$ and $\Lambda^{*}, \widehat{\Pi}(\tau)$ in Eq. (29) is not diagonal in the creation and annihilation operators. If it were, we could easily calculate the expectation values of products of creation and annihilation operators, hence of operators quadratic in the field, using standard methods. We thus look for a suitable Bogolyubov transformation that diagonalizes $\widehat{\Pi}(\tau)$,

$$
\begin{aligned}
& \widehat{\xi}_{\mathrm{p}}^{\dagger}(\tau)=A(\mathrm{p}, \tau) \widehat{b}_{\mathrm{p}}^{\dagger}-B(\mathrm{p}, \tau) \widehat{b}_{-\mathrm{p}}, \\
& \widehat{\xi}_{\mathrm{p}}(\tau)=A^{*}(\mathrm{p}, \tau) \widehat{b}_{\mathrm{p}}-B^{*}(\mathrm{p}, \tau) \widehat{b}_{-\mathrm{p}}^{\dagger},
\end{aligned}
$$

where $A$ and $B$ are complex functions to be determined. We require $\widehat{\xi}_{p}^{\dagger}$ and $\widehat{\xi}_{p}$ to fulfill the usual algebra:

$$
\begin{aligned}
& {\left[\widehat{\xi}_{\mathrm{p}}(\tau), \widehat{\xi}_{\mathrm{p}^{\prime}}^{\dagger}(\tau)\right]=\delta^{2}\left(\mathbf{p}_{\mathrm{T}}-\mathbf{p}_{\mathrm{T}}^{\prime}\right) \delta\left(\mu-\mu^{\prime}\right),} \\
& {\left[\widehat{\xi}_{\mathrm{p}}(\tau), \widehat{\xi}_{\mathrm{p}^{\prime}}(\tau)\right]=0=\left[\widehat{\xi}_{\mathrm{p}}^{\dagger}(\tau), \widehat{\xi}_{\mathrm{p}^{\prime}}^{\dagger}(\tau)\right],}
\end{aligned}
$$

so that, by enforcing the commutation relations (22), we find respectively

$$
\begin{aligned}
& \left(|A(\mathrm{p}, \tau)|^{2}-|B(\mathrm{p}, \tau)|^{2}\right) \delta^{2}\left(\mathbf{p}_{\mathrm{T}}-\mathbf{p}_{\mathrm{T}}^{\prime}\right) \delta\left(\mu-\mu^{\prime}\right)=\delta^{2}\left(\mathbf{p}_{\mathrm{T}}-\mathbf{p}_{\mathrm{T}}^{\prime}\right) \delta\left(\mu-\mu^{\prime}\right), \\
& {\left[A^{*}(-\mathrm{p}, \tau) B^{*}(\mathrm{p}, \tau)-A^{*}(\mathrm{p}, \tau) B^{*}(-\mathrm{p}, \tau)\right] \delta^{2}\left(\mathbf{p}_{\mathrm{T}}+\mathbf{p}_{\mathrm{T}}^{\prime}\right) \delta\left(\mu+\mu^{\prime}\right)=0} \\
& {[A(\mathrm{p}, \tau) B(-\mathrm{p}, \tau)-A(-\mathrm{p}, \tau) B(\mathrm{p}, \tau)] \delta^{2}\left(\mathbf{p}_{\mathrm{T}}+\mathbf{p}_{\mathrm{T}}^{\prime}\right) \delta\left(\mu+\mu^{\prime}\right)=0 .}
\end{aligned}
$$


The above equation is fulfilled if

$$
\begin{gathered}
A(\mathrm{p}, \tau)=A(-\mathrm{p}, \tau), \quad B(\mathrm{p}, \tau)=B(-\mathrm{p}, \tau), \\
|A(\mathrm{p}, \tau)|^{2}-|B(\mathrm{p}, \tau)|^{2}=1,
\end{gathered}
$$

so we can set

$$
\begin{aligned}
& A(\mathrm{p}, \tau)=\cosh \theta(\mathrm{p}, \tau) \mathrm{e}^{i \chi_{A}(\mathrm{p}, \tau)}, \\
& B(\mathrm{p}, \tau)=\sinh \theta(\mathrm{p}, \tau) \mathrm{e}^{i \chi_{B}(\mathrm{p}, \tau)} .
\end{aligned}
$$

The conditions (37) make it easier to invert Eq. (35):

$$
\begin{aligned}
& \widehat{b}_{\mathrm{p}}=A(\mathrm{p}, \tau) \widehat{\xi}_{\mathrm{p}}(\tau)+B^{*}(\mathrm{p}, \tau) \widehat{\xi}_{-\mathrm{p}}^{\dagger}(\tau), \\
& \widehat{b}_{\mathrm{p}}^{\dagger}=A^{*}(\mathrm{p}, \tau) \widehat{\xi}_{\mathrm{p}}^{\dagger}(\tau)+B(\mathrm{p}, \tau) \widehat{\xi}_{-\mathrm{p}}(\tau) .
\end{aligned}
$$

Plugging Eq. (39) into Eq. (29) we obtain

$$
\begin{aligned}
\widehat{\Pi}(\tau)= & \int \mathrm{d}^{2} p_{T} \mathrm{~d} \mu \frac{\omega}{2}\left\{\left[K\left(|A|^{2}+|B|^{2}\right)\right.\right. \\
& \left.+\Lambda A B^{*}+\Lambda^{*} A^{*} B\right]\left(\widehat{\xi}_{p} \widehat{\xi}_{p}^{\dagger}+\widehat{\xi}_{p}^{\dagger} \widehat{\xi}_{\mathrm{p}}\right) \\
& +\left(2 K A B+\Lambda A^{2}+\Lambda^{*} B^{2}\right) \widehat{\xi}_{\mathrm{p}} \widehat{\xi}_{-\mathrm{p}} \\
& \left.+\left(2 K A^{*} B^{*}+\Lambda^{*} A^{* 2}+\Lambda B^{* 2}\right) \widehat{\xi}_{\mathrm{p}}^{\dagger} \widehat{\xi}_{-\mathrm{p}}^{\dagger}\right\},
\end{aligned}
$$

where we used the invariance of the integral under reflections $p \mapsto-p$. In order to make $\widehat{\Pi}(\tau)$ diagonal, the second line of Eq. (40) must vanish:

$$
2 K A B+\Lambda A^{2}+\Lambda^{*} B^{2}=0
$$

(the other equation is just the complex conjugate). This can be rewritten by using Eqs. (34) and (38):

$$
\begin{aligned}
& \cosh 2 \Theta \sinh 2 \theta \mathrm{e}^{i\left(\chi_{A}+\chi_{B}\right)}+\sinh 2 \Theta \cosh ^{2} \theta \mathrm{e}^{i\left(\chi+2 \chi_{A}\right)} \\
& +\sinh 2 \Theta \sinh ^{2} \theta \mathrm{e}^{i\left(2 \chi_{B}-\chi\right)}=0
\end{aligned}
$$

the solution of which is

$$
\chi_{B}-\chi_{A}=\chi, \quad \theta=-\Theta .
$$

We can then set $\chi_{A}=0$ and find $A, B$ fulfilling the Bogolyubov relations (35)

$$
A=\cosh \Theta, \quad B=-\sinh \Theta \mathrm{e}^{i \chi},
$$

from which follows, by using Eq. (34)

$$
\begin{aligned}
& K\left(|A|^{2}+|B|^{2}\right)+\Lambda A B^{*}+\Lambda^{*} A^{*} B \\
& \quad=\cosh ^{2} 2 \Theta-2 \operatorname{Re}\left(\sinh 2 \Theta \mathrm{e}^{i \chi} \cosh \Theta \sinh \Theta \mathrm{e}^{-i \chi}\right)=1 .
\end{aligned}
$$

With these solutions, Eq. (39) becomes $\widehat{b}_{\mathrm{p}}=\cosh \Theta(\mathrm{p}, \tau) \widehat{\xi}_{\mathrm{p}}(\tau)-\sinh \Theta(\mathrm{p}, \tau) \mathrm{e}^{-i \chi \widehat{\xi}_{-\mathrm{p}}^{\dagger}}(\tau)$,

$\widehat{b}_{\mathrm{p}}^{\dagger}=\cosh \Theta(\mathrm{p}, \tau) \widehat{\xi}_{\mathrm{p}}^{\dagger}(\tau)-\sinh \Theta(\mathrm{p}, \tau) \mathrm{e}^{i \chi \widehat{\xi}_{-\mathrm{p}}(\tau),}$

and the operator (40):

$$
\begin{aligned}
\widehat{\Pi}(\tau) & =\int \mathrm{d}^{2} p_{T} \mathrm{~d} \mu \frac{\omega}{2}\left(\widehat{\xi}_{\mathrm{p}}(\tau) \widehat{\xi}_{\mathrm{p}}^{\dagger}(\tau)+\widehat{\xi}_{\mathrm{p}}^{\dagger}(\tau) \widehat{\xi}_{\mathrm{p}}(\tau)\right) \\
& =\int \mathrm{d}^{2} p_{T} \mathrm{~d} \mu \omega\left(\widehat{\xi}_{\mathrm{p}}^{\dagger}(\tau) \widehat{\xi}_{\mathrm{p}}(\tau)+\frac{1}{2}\right),
\end{aligned}
$$

where in the last equality we have used the commutation relations (36).

\section{A. Discusssion}

The nontrivial Bogoliubov transformation (42) between different sets of creation and annihilation operators is reminiscent of the Unruh effect [24]. However, we are facing essentially different physics here; as it has been pointed out, the relation (23) between plane-wave creation operators and the creation operators appearing in the field expansion in curvilinear coordinates does not mix creation and annihilation operators. In other words, unlike in the Unruh effect, the observers associated with Milne coordinates (defined by $\eta=\mathbf{x}_{\mathrm{T}}=$ const) (moving with zero acceleration as implied by the velocity field $u$ in (17), count the same particles as the conventionally fixed inertial observer.

In fact, the Bogolyubov transformation (42) stems from the somewhat unexpected form of the local thermodynamic equilibrium operator $\widehat{\Pi}$ in Eq. (29) involving quadratic combinations of two annihilation and two creation operators, unlike the Hamiltonian in global-equilibrium thermal field theory. We thus have a concrete situation where the vacuum $\left|0_{\tau}\right\rangle$, which is the lowest-lying eigenvector of $\widehat{\Pi}(\tau)$ annihilated by all $\widehat{\xi}_{\mathrm{p}}(\tau)$ 's,

$$
\widehat{\xi}_{\mathrm{p}}(\tau)\left|0_{\tau}\right\rangle=0,
$$

is different from the Minkowski vacuum $\left|0_{M}\right\rangle$, which is annihilated by the $\widehat{b}_{\mathrm{p}}$, as envisioned in Sec. III A. The full expression of the vacuum $\left|0_{\tau}\right\rangle$ can be obtained from the coefficients in Eq. (42) with known methods [22] and reads

$$
\begin{aligned}
\left|0_{\tau}\right\rangle= & \prod_{\mathrm{p}} \frac{1}{|\cosh \Theta(\mathrm{p}, \tau)|^{1 / 2}} \\
& \times \exp \left[-\frac{1}{2} \tanh \Theta(\mathrm{p}, \tau) \mathrm{e}^{-i \chi(\mathrm{p}, \tau)} \widehat{b}_{\mathrm{p}}^{\dagger} \hat{b}_{-\mathrm{p}}^{\dagger}\right]\left|0_{M}\right\rangle .
\end{aligned}
$$

With $\widehat{\Pi}$ diagonal in Eq. (43), we can readily obtain the expectation values of products of creation and annihilation operators in local thermodynamic equilibrium. The form (43) is essentially the same as the equilibrium Hamiltonian operator of the free field with the replacements $\mu \rightarrow p_{z}$ and $\omega \rightarrow \varepsilon$. We thus have 


$$
\begin{aligned}
\left\langle\widehat{\xi}_{\mathrm{p}}^{\dagger}(\tau) \widehat{\xi}_{\mathrm{p}^{\prime}}(\tau)\right\rangle_{\mathrm{LE}} & =n_{\mathrm{B}}(\mathrm{p}, \tau) \delta^{2}\left(\mathbf{p}_{\mathrm{T}}-\mathbf{p}_{\mathrm{T}}^{\prime}\right) \delta\left(\mu-\mu^{\prime}\right), \\
\left\langle\widehat{\xi}_{\mathrm{p}}(\tau) \widehat{\xi}_{\mathrm{p}^{\prime}}^{\dagger}(\tau)\right\rangle_{\mathrm{LE}} & =\left[n_{\mathrm{B}}(\mathrm{p}, \tau)+1\right] \delta^{2}\left(\mathbf{p}_{\mathrm{T}}-\mathbf{p}_{\mathrm{T}}^{\prime}\right) \delta\left(\mu-\mu^{\prime}\right), \\
\left\langle\widehat{\xi}_{\mathrm{p}}(\tau) \widehat{\xi}_{\mathrm{p}^{\prime}}(\tau)\right\rangle_{\mathrm{LE}} & =0=\left\langle\widehat{\xi}_{\mathrm{p}}^{\dagger}(\tau) \widehat{\xi}_{\mathrm{p}^{\prime}}^{\dagger}(\tau)\right\rangle_{\mathrm{LE}},
\end{aligned}
$$

where $\langle\cdot\rangle_{\mathrm{LE}}$ stands for $\operatorname{Tr}\left(\widehat{\rho}_{\mathrm{LE}} \cdot\right)$ and $n_{\mathrm{B}}$ is the Bose-Einstein distribution function:

$$
n_{\mathrm{B}}(\mathrm{p}, \tau)=\frac{1}{\mathrm{e}^{\omega(\tau) / T(\tau)}-1},
$$

with $\omega(\tau)$ given by Eq. (27).

It is important to emphasize that Eq. (46) is by no means a density of particles as usually in Minkowski space-time. Equation (46) accounts for the mean number of excitations of the $\widehat{\xi}_{p}^{\dagger}(\tau)$ operator, which is not the mean number of excitations of the Minkowski vacuum as expressed by the $\widehat{a}(p)$ 's or $\widehat{b}_{\mathrm{p}}$ 's. Indeed, the expectation values of the various combinations can be found by means of Eq. (42) and Eq. (45):

$$
\begin{aligned}
&\left\langle\widehat{b}_{\mathrm{p}} \widehat{b}_{\mathrm{p}^{\prime}}\right\rangle_{\mathrm{LE}}=-\frac{1}{2} \sinh (2 \Theta) \mathrm{e}^{-i \chi}\left(2 n_{\mathrm{B}}+1\right) \delta^{2}\left(\mathbf{p}_{\mathrm{T}}-\mathbf{p}_{\mathrm{T}}^{\prime}\right) \delta\left(\mu-\mu^{\prime}\right), \\
&\left\langle\widehat{b}_{\mathrm{p}}^{\dagger} \widehat{b}_{\mathrm{p}^{\prime}}^{\dagger}\right\rangle_{\mathrm{LE}}=-\frac{1}{2} \sinh (2 \Theta) \mathrm{e}^{i \chi}\left(2 n_{\mathrm{B}}+1\right) \delta^{2}\left(\mathbf{p}_{\mathrm{T}}-\mathbf{p}_{\mathrm{T}}^{\prime}\right) \delta\left(\mu-\mu^{\prime}\right), \\
&\left\langle\widehat{b}_{\mathrm{p}} \widehat{b}_{\mathrm{p}^{\prime}}^{\dagger}\right\rangle_{\mathrm{LE}}=\left[n_{\mathrm{B}} \cosh (2 \Theta)+\cosh ^{2} \Theta\right] \delta^{2}\left(\mathbf{p}_{\mathrm{T}}-\mathbf{p}_{\mathrm{T}}^{\prime}\right) \delta\left(\mu-\mu^{\prime}\right), \\
&\left\langle\widehat{b}_{\mathrm{p}}^{\dagger} \widehat{b}_{\mathrm{p}^{\prime}}\right\rangle_{\mathrm{LE}}=\left[n_{\mathrm{B}} \cosh (2 \Theta)+\sinh ^{2} \Theta\right] \delta^{2}\left(\mathbf{p}_{\mathrm{T}}-\mathbf{p}_{\mathrm{T}}^{\prime}\right) \delta\left(\mu-\mu^{\prime}\right) .
\end{aligned}
$$

As is clear from Eq. (47), field vacuum effects are encoded in a nonvanishing value of the angle $\Theta(p, \tau)$, which is both a function of the modes and of the Milne time $\tau$ and whose value can be determined through the relations (34). We are now in a position to calculate the expectation values of all operators which are quadratic in the field.

\section{THE STRESS-ENERGY TENSOR AND ITS RENORMALIZATION}

We now come to the main point of this work, namely the determination of the stress-energy tensor. We start by calculating it in local thermodynamic equilibrium.

\section{A. Local thermodynamic equilibrium}

As the symmetries of $\widehat{\rho}_{\mathrm{LE}}$ are the same as $\widehat{\rho}$ (see the discussion in Sec. II) the structure must be the same as in Eq. (15):

$$
\begin{aligned}
\operatorname{Tr}\left(\widehat{\rho}_{\mathrm{LE}} \widehat{T}^{\mu \nu}\right)= & \left\langle\widehat{T}^{\mu \nu}\right\rangle_{\mathrm{LE}}=\mathcal{E}(\tau)_{\mathrm{LE}} u^{\mu} u^{\nu} \\
& +\mathcal{P}_{\mathrm{T}}(\tau)_{\mathrm{LE}}\left(\widehat{i}^{\wedge} \hat{i}^{\nu}+\widehat{j}^{\mu} \widehat{j}^{\nu}\right)+\mathcal{P}_{\mathrm{L}}(\tau)_{\mathrm{LE}} \widehat{\eta}^{\mu} \widehat{\eta}^{\nu},
\end{aligned}
$$

Hence, by using Eq. (28) with the expansion (21) we obtain

$$
\begin{aligned}
\mathcal{E}(\tau)_{\mathrm{LE}}= & \left\langle\widehat{T}_{C}^{\mu \nu}\right\rangle_{\mathrm{LE}} u_{\mu} u_{\nu}=\int \frac{d^{2} p_{T} \mathrm{~d} \mu d^{2} \mathrm{p}_{\mathrm{T}}^{\prime} \mathrm{d} \mu^{\prime}}{4(4 \pi)^{2}}\left(\left\{\left[\partial_{\tau} h(\mathbf{p}, \tau)\right]\left[\partial_{\tau} h\left(\mathbf{p}^{\prime}, \tau\right)\right]-\left(p_{x} p_{x}^{\prime}+p_{y} p_{y}^{\prime}+\frac{1}{\tau^{2}} \mu \mu^{\prime}-m^{2}\right) h(\mathbf{p}, \tau) h\left(\mathbf{p}^{\prime}, \tau\right)\right\}\right. \\
& \times \mathrm{e}^{i\left[\left(\mathbf{p}_{\mathrm{T}}+\mathbf{p}_{\mathrm{T}}^{\prime}\right) \cdot \mathbf{x}_{\mathrm{T}}+\left(\mu+\mu^{\prime}\right) \eta\right]}\left\langle\widehat{b}_{\mathrm{p}} \widehat{b}_{\mathbf{p}^{\prime}}\right\rangle_{\mathrm{LE}} \\
& +\left\{\left[\partial_{\tau} h(\mathbf{p}, \tau)\right]\left[\partial_{\tau} h^{*}\left(\mathbf{p}^{\prime}, \tau\right)\right]+\left(p_{x} p_{x}^{\prime}+p_{y} p_{y}^{\prime}+\frac{1}{\tau^{2}} \mu \mu^{\prime}+m^{2}\right) h(\mathbf{p}, \tau) h^{*}\left(\mathbf{p}^{\prime}, \tau\right)\right\} \mathrm{e}^{i\left[\left(\mathbf{p}_{\mathrm{T}}-\mathbf{p}_{\mathrm{T}}^{\prime}\right) \cdot \mathbf{x}_{\mathrm{T}}+\left(\mu-\mu^{\prime}\right) \eta\right]}\left\langle\widehat{b}_{\mathbf{p}} \widehat{b}_{\mathbf{p}^{\prime}}^{\dagger}\right\rangle_{\mathrm{LE}} \\
& +\left\{\left[\partial_{\tau} h^{*}(\mathbf{p}, \tau)\right]\left[\partial_{\tau} h\left(\mathbf{p}^{\prime}, \tau\right)\right]+\left(p_{x} p_{x}^{\prime}+p_{y} p_{y}^{\prime}+\frac{1}{\tau^{2}} \mu \mu^{\prime}+m^{2}\right) h^{*}(\mathbf{p}, \tau) h\left(\mathbf{p}^{\prime}, \tau\right)\right\} \mathrm{e}^{-i\left[\left(\mathbf{p}_{\mathrm{T}}-\mathbf{p}_{\mathrm{T}}^{\prime}\right) \cdot \mathbf{x}_{\mathrm{T}}+\left(\mu-\mu^{\prime}\right) \eta\right]}\left\langle\widehat{b}_{\mathrm{p}}^{\dagger} \widehat{b}_{\mathbf{p}^{\prime}}\right\rangle_{\mathrm{LE}} \\
& \left.+\left\{\left[\partial_{\tau} h^{*}(\mathbf{p}, \tau)\right]\left[\partial_{\tau} h^{*}\left(\mathbf{p}^{\prime}, \tau\right)\right]-\left(p_{x} p_{x}^{\prime}+p_{y} p_{y}^{\prime}+\frac{1}{\tau^{2}} \mu \mu^{\prime}-m^{2}\right) h^{*}(\mathbf{p}, \tau) h^{*}\left(\mathbf{p}^{\prime}, \tau\right)\right\} \mathrm{e}^{\left.-i\left[\mathbf{p}_{\mathrm{T}}+\mathbf{p}_{\mathrm{T}}^{\prime}\right) \cdot \mathbf{x}_{\mathrm{T}}+\left(\mu+\mu^{\prime}\right) \eta\right]}\left\langle\widehat{b}_{\mathrm{p}}^{\dagger} \widehat{b}_{\mathbf{p}^{\prime}}^{\dagger}\right\rangle_{\mathrm{LE}}\right) .
\end{aligned}
$$

Plugging the relations (47) into Eq. (49) we obtain

$$
\mathcal{E}(\tau)_{\mathrm{LE}}=\frac{1}{4(4 \pi)^{2}} \int \mathrm{d}^{2} \mathrm{p}_{\mathrm{T}} \mathrm{d} \mu\left\{-\frac{1}{2}\left[\left(\partial_{\tau} h\right)^{2}+\omega^{2} h^{2}\right]\left(2 n_{\mathrm{B}}+1\right) \sinh (2 \Theta) \mathrm{e}^{-i \chi}+\text { c.c. }+\left(\left|\partial_{\tau} h\right|^{2}+\omega^{2}|h|^{2}\right)\left(2 n_{\mathrm{B}}+1\right) \cosh (2 \Theta)\right\}
$$

and, by using Eqs. (30), (31), and (34), we get

$$
\mathcal{E}(\tau)_{\mathrm{LE}}=\frac{1}{16 \pi^{3} \tau} \int \mathrm{d}^{2} \mathrm{p}_{\mathrm{T}} \mathrm{d} \mu \omega\left[\left(2 n_{\mathrm{B}}+1\right) \cosh ^{2}(2 \Theta)-\sinh ^{2}(2 \Theta)\left(2 n_{\mathrm{B}}+1\right)\right]=\frac{1}{(2 \pi)^{3} \tau} \int \mathrm{d}^{2} \mathrm{p}_{\mathrm{T}} \mathrm{d} \mu \omega\left(n_{\mathrm{B}}+\frac{1}{2}\right) .
$$


The longitudinal and transverse pressures can be worked out in a similar fashion: the Wronskian of the Hankel function is again recovered and the expressions greatly simplify. One obtains

$$
\begin{aligned}
& \mathcal{P}_{\mathrm{T}}(\tau)_{\mathrm{LE}}=\frac{1}{(2 \pi)^{3} \tau} \int \mathrm{d}^{2} \mathrm{p}_{\mathrm{T}} \mathrm{d} \mu \frac{\left|\mathbf{p}_{\mathrm{T}}\right|^{2}}{2}\left(n_{\mathrm{B}}+\frac{1}{2}\right), \\
& \mathcal{P}_{\mathrm{L}}(\tau)_{\mathrm{LE}}=\frac{1}{(2 \pi)^{3} \tau} \int \mathrm{d}^{2} \mathrm{p}_{\mathrm{T}} \mathrm{d} \mu \frac{\mu^{2}}{\tau^{2}}\left(n_{\mathrm{B}}+\frac{1}{2}\right) .
\end{aligned}
$$

Equations (50) and (51) can be written in a compact fashion by introducing the functions

$$
\begin{aligned}
& K_{\gamma}(\mathrm{p}, \tau)=\frac{\pi \tau}{4 \omega}\left[\left|\partial_{\tau} h(\mathrm{p}, \tau)\right|^{2}+\gamma(\mathrm{p}, \tau)|h(\mathrm{p}, \tau)|^{2}\right], \\
& \Lambda_{\gamma}(\mathrm{p}, \tau)=\frac{\pi \tau}{4 \omega}\left\{\left[\partial_{\tau} h(\mathrm{p}, \tau)\right]^{2}+\gamma(\mathrm{p}, \tau)[h(\mathrm{p}, \tau)]^{2}\right\},
\end{aligned}
$$

where $\gamma$ is defined as

$$
\gamma(\mathbf{p}, \tau)= \begin{cases}\omega^{2}(\mathbf{p}, \tau)=m_{\mathrm{T}}^{2}+\frac{\mu^{2}}{\tau^{2}}, & \text { for } \mathcal{E}(\tau)_{\mathrm{LE}}, \\ -m_{\mathrm{L}}^{2} \equiv-\frac{\mu^{2}}{\tau^{2}}-m^{2}, & \text { for } \mathcal{P}_{\mathrm{T}}(\tau)_{\mathrm{LE}}, \\ -m_{\mathrm{T}}^{2}+\frac{\mu^{2}}{\tau^{2}}, & \text { for } \mathcal{P}_{\mathrm{L}}(\tau)_{\mathrm{LE}} .\end{cases}
$$

Thanks to the Wronskian of the Hankel functions, they satisfy the relation

$$
K_{\gamma}^{2}(\mathrm{p}, \tau)-\left|\Lambda_{\gamma}(\mathrm{p}, \tau)\right|^{2}=\frac{\gamma(\mathrm{p}, \tau)}{\omega^{2}(\mathrm{p}, \tau)} .
$$

With this in mind, and setting $\Gamma_{\gamma}=\left\{\mathcal{E}, \mathcal{P}_{\mathrm{T}}, \mathcal{P}_{\mathrm{L}}\right\}$, we have for the thermodynamic function of the stress-energy tensor

$$
\begin{aligned}
\Gamma_{\gamma}(\tau)_{\mathrm{LE}}= & \int \frac{d^{2} \mathrm{p}_{\mathrm{T}} \mathrm{d} \mu}{(2 \pi)^{3} \tau} \omega(\mathrm{p}, \tau)\left[K_{\gamma}(\mathrm{p}, \tau) K(\mathrm{p}, \tau)\right. \\
& \left.-\operatorname{Re}\left(\Lambda_{\gamma}(\mathrm{p}, \tau) \Lambda^{*}(\mathrm{p}, \tau)\right)\right]\left(n_{\mathrm{B}}(\mathrm{p}, \tau)+\frac{1}{2}\right),
\end{aligned}
$$

where the combination in square brackets reads

$K_{\gamma}(\mathrm{p}, \tau) K(\mathrm{p}, \tau)-\operatorname{Re}\left(\Lambda \gamma(\mathrm{p}, \tau) \Lambda^{*}(\mathrm{p}, \tau)\right)=\frac{\omega^{2}(\mathrm{p}, \tau)+\gamma(\mathrm{p}, \tau)}{2 \omega^{2}(\mathrm{p}, \tau)}$,

hence

$\Gamma_{\gamma}(\tau)_{\mathrm{LE}}=\int \frac{d^{2} \mathrm{p}_{\mathrm{T}} \mathrm{d} \mu}{(2 \pi)^{3} \tau \omega(\mathrm{p}, \tau)} \frac{\omega^{2}(\mathrm{p}, \tau)+\gamma(\mathrm{p}, \tau)}{2}\left[n_{\mathrm{B}}(\mathrm{p}, \tau)+\frac{1}{2}\right]$.

The above integrals can be written in a familiar form by changing the integration variable to $p_{z}=\mu / \tau$. This implies

$$
\omega(\mathbf{p}, \tau)=\sqrt{\left|\mathbf{p}_{\mathrm{T}}\right|^{2}+\frac{\mu^{2}}{\tau^{2}}+m^{2}}=\sqrt{\mathrm{p}_{x}^{2}+\mathrm{p}_{y}^{2}+\mathrm{p}_{z}^{2}+m^{2}}=\varepsilon,
$$

which is just the on-shell energy, and

$$
\mathrm{d}^{2} \mathrm{p}_{\mathrm{T}} \frac{\mathrm{d} \mu}{\tau}=\mathrm{dp}_{x} \mathrm{dp}_{y} \mathrm{dp}_{z}
$$

In turn, the distribution $n_{\mathrm{B}}(\mathrm{p}, \tau)$ becomes the energydependent Bose-Einstein phase-space distribution $n_{\mathrm{B}}(\varepsilon, T(\tau))$. Hence, the first term of the energy density (50) as well as the transverse and longitudinal pressures (51) can be written as the familiar momentum integrals of the relativistic uncharged Bose gas. Altogether, the unrenormalized stress-energy tensor in local equilibrium reads:

$$
\operatorname{Tr}\left[\widehat{\rho}_{\mathrm{LE}} \widehat{T}^{\mu \nu}(x)\right]=\int \frac{\mathrm{d}^{3} p}{\varepsilon} p^{\mu} p^{\nu}\left[\frac{1}{\mathrm{e}^{\beta(x) \cdot p}-1}+\frac{1}{2}\right],
$$

where $\beta$ is the four-temperature in Eq. (12). Hence, the thermodynamic functions $\Gamma_{\gamma}$ are just the familiar functions of $T(\tau)$ as for the ideal relativistic gas. In particular, the transverse and the longitudinal pressures are in fact identical, namely

$$
\mathcal{P}_{\mathrm{T}}(\tau)_{\mathrm{LE}}=\mathcal{P}_{\mathrm{L}}(\tau)_{\mathrm{LE}} \equiv \mathcal{P}(\tau)_{\mathrm{LE}}
$$

\section{B. Actual stress-energy tensor}

The actual (unrenormalized) expectation value of the stress-energy tensor can be calculated by using the density operator (16), that is,

$$
\operatorname{Tr}\left[\widehat{\rho} \widehat{T}^{\mu \nu}(x)\right]=\frac{1}{Z} \operatorname{Tr}\left\{\exp \left[-\widehat{\Pi}\left(\tau_{0}\right) / T\left(\tau_{0}\right)\right] \widehat{T}^{\mu \nu}(x)\right\} .
$$

Symmetries dictate that its form is given by Eq. (15), so we need to determine the three functions $\Gamma_{\gamma}$. It is readily found that the same expression as in Eq. (49) is obtained, with the simple replacement of the local-equilibrium values of the quadratic combinations of $\widehat{b}_{\mathrm{p}}$ and $\widehat{b}_{\mathrm{p}}^{\dagger}$ with their actual expectation values, for instance,

$$
\left\langle\widehat{b}_{\mathrm{p}}^{\dagger} \widehat{b}_{\mathrm{p}}\right\rangle=\frac{1}{Z} \operatorname{Tr}\left\{\exp \left[-\widehat{\Pi}\left(\tau_{0}\right) / T\left(\tau_{0}\right)\right] \widehat{b}_{\mathrm{p}}^{\dagger} \widehat{b}_{\mathrm{p}}\right\} .
$$

The calculation of the above expression is most easily done by using the formulas (42) at time $\tau_{0}$, i.e., expressing the constant $\widehat{b}_{\mathbf{p}}$ 's as functions of the operators diagonalizing $\widehat{\Pi}\left(\tau_{0}\right)$ instead of $\widehat{\Pi}(\tau)$. We thus get the same formulas as Eq. (47), with $\tau$ replaced by $\tau_{0}$ : 


$$
\begin{aligned}
& \left\langle\widehat{b}_{\mathrm{p}} \widehat{b}_{\mathrm{p}^{\prime}}\right\rangle=-\frac{1}{2} \sinh \left[2 \Theta\left(\tau_{0}\right)\right] \mathrm{e}^{-i \chi\left(\tau_{0}\right)}\left[2 n_{\mathrm{B}}\left(\tau_{0}\right)+1\right] \delta^{2}\left(\mathbf{p}_{\mathrm{T}}-\mathbf{p}_{\mathrm{T}}^{\prime}\right) \delta\left(\mu-\mu^{\prime}\right), \\
& \left\langle\widehat{b}_{\mathrm{p}}^{\dagger} \widehat{b}_{\mathrm{p}^{\prime}}^{\dagger}\right\rangle=-\frac{1}{2} \sinh \left[2 \Theta\left(\tau_{0}\right)\right] \mathrm{e}^{i \chi\left(\tau_{0}\right)}\left[2 n_{\mathrm{B}}\left(\tau_{0}\right)+1\right] \delta^{2}\left(\mathbf{p}_{\mathrm{T}}-\mathbf{p}_{\mathrm{T}}^{\prime}\right) \delta\left(\mu-\mu^{\prime}\right), \\
& \left\langle\widehat{b}_{\mathrm{p}} \widehat{b}_{\mathrm{p}^{\prime}}^{\dagger}\right\rangle=\left\{n_{\mathrm{B}}\left(\tau_{0}\right) \cosh \left[2 \Theta\left(\tau_{0}\right)\right]+\cosh ^{2} \Theta\left(\tau_{0}\right)\right\} \delta^{2}\left(\mathbf{p}_{\mathrm{T}}-\mathbf{p}_{\mathrm{T}}^{\prime}\right) \delta\left(\mu-\mu^{\prime}\right), \\
& \left\langle\widehat{b}_{\mathrm{p}}^{\dagger} \widehat{b}_{\mathrm{p}^{\prime}}\right\rangle=\left\{n_{\mathrm{B}}\left(\tau_{0}\right) \cosh \left[2 \Theta\left(\tau_{0}\right)\right]+\sinh ^{2} \Theta\left(\tau_{0}\right)\right\} \delta^{2}\left(\mathbf{p}_{\mathrm{T}}-\mathbf{p}_{\mathrm{T}}^{\prime}\right) \delta\left(\mu-\mu^{\prime}\right) .
\end{aligned}
$$

We note in passing that, as expected, the expectation value of excitations of the Minkowski vacuum, described by $\left\langle\widehat{b}_{\mathrm{p}}^{\dagger} \widehat{b}_{\mathrm{p}}\right\rangle$ for each mode, is constant in time, the density operator being fixed and the operators $\widehat{b}_{\mathrm{p}}$ being time independent by construction. The mean number of particles with momentum $p$ can be obtained by using Eq. (23):

$$
\begin{aligned}
\left\langle\widehat{a}^{\dagger}(p) \widehat{a}\left(p^{\prime}\right)\right\rangle= & \frac{1}{2 \pi m_{\mathrm{T}}} \frac{1}{\sqrt{\cosh y \cosh y^{\prime}}} \\
& \times \int_{-\infty}^{+\infty} \mathrm{d} \mu \mathrm{e}^{-i \mu\left(y-y^{\prime}\right)}\left\langle\widehat{b}_{\mathbf{p}_{T}, \mu}^{\dagger} \widehat{b}_{\mathbf{p}_{T}^{\prime}, \mu}\right\rangle,
\end{aligned}
$$

where $y$ is the rapidity.

Now, by taking advantage of the right-hand side of Eq. (49), and by using Eq. (58), as well as Eqs. (30), (31), and (34), it can be shown that

$$
\begin{aligned}
\mathcal{E}(\tau)= & \frac{1}{Z} \operatorname{Tr}\left\{\exp \left[-\widehat{\Pi}\left(\tau_{0}\right) / T\left(\tau_{0}\right)\right] \widehat{T}^{\mu \nu}\right\} u_{\mu} u_{\nu} \\
= & \frac{1}{(2 \pi)^{3} \tau} \int d^{2} \mathrm{p}_{\mathrm{T}} \mathrm{d} \mu \omega(\tau)\left\{K(\tau) K\left(\tau_{0}\right)\right. \\
& \left.-\operatorname{Re}\left[\Lambda(\tau) \Lambda^{*}\left(\tau_{0}\right)\right]\right\}\left[n_{\mathrm{B}}\left(\tau_{0}\right)+\frac{1}{2}\right] .
\end{aligned}
$$

The pressures can be derived likewise and we finally have

$$
\begin{aligned}
\Gamma_{\gamma}(\tau)= & \frac{1}{(2 \pi)^{3} \tau} \int d^{2} \mathrm{p}_{\mathrm{T}} \mathrm{d} \mu \omega(\tau)\left\{K_{\gamma}(\tau) K\left(\tau_{0}\right)\right. \\
& \left.-\operatorname{Re}\left[\Lambda_{\gamma}(\tau) \Lambda^{*}\left(\tau_{0}\right)\right]\right\}\left[n_{\mathrm{B}}\left(\tau_{0}\right)+\frac{1}{2}\right]
\end{aligned}
$$

Of course, at the time $\tau=\tau_{0}$ we recover the local thermodynamic equilibrium expression (55), as required by construction. However, at later times $\tau>\tau_{0}$ the stressenergy tensor differs from the local equilibrium form. Indeed, since we are dealing with a free field, one expects to find the same expression as for the free-streaming solution of the Boltzmann equation in Milne coordinates, see Appendix A. However, there are quantum corrections due to the vacuum subtraction.

\section{Renormalization and comparison with classical limits}

The expressions found include divergent terms, both in the stress-energy tensor in local equilibrium (55) and the actual one (60). As we have seen in Sec. III A, in order to fulfill the continuity equation, the stress-energy tensor should be renormalized by subtracting a vacuum expectation value (VEV) with a constant vacuum: either with respect to the Minkowskian vacuum $\left|0_{M}\right\rangle$, like in Eq. (19), or with respect to the vacuum $\left|0_{\tau_{0}}\right\rangle$ of the operator $\widehat{\Pi}\left(\tau_{0}\right)$, like in Eq. (20).

The Minkowski VEV of the stress-energy tensor is calculated in Appendix B. For the stress-energy tensor it is found that

$$
\mathcal{E}_{M} \equiv\left\langle 0_{M}\left|\widehat{T}^{\mu \nu} u_{\mu} u_{\nu}\right| 0_{M}\right\rangle=\frac{1}{(2 \pi)^{3} \tau} \int d^{2} \mathrm{p}_{\mathrm{T}} \mathrm{d} \mu \omega(\tau) \frac{K(\tau)}{2},
$$

and the renormalized energy density is then

$$
\begin{aligned}
\mathcal{E}(\tau)_{\text {ren }}= & \frac{1}{(2 \pi)^{3} \tau} \int d^{2} \mathrm{p}_{\mathrm{T}} \mathrm{d} \mu \omega(\tau)\left(\left\{K(\tau) K\left(\tau_{0}\right)\right.\right. \\
& \left.\left.-\operatorname{Re}\left[\Lambda(\tau) \Lambda^{*}\left(\tau_{0}\right)\right]\right\}\left[n_{\mathrm{B}}\left(\tau_{0}\right)+\frac{1}{2}\right]-\frac{1}{2} K(\tau)\right) .
\end{aligned}
$$

The main drawback of this expression is that it is still divergent. This is most easily seen at $\tau=\tau_{0}$ where

$$
\begin{aligned}
\mathcal{E}\left(\tau_{0}\right)_{\text {ren }}= & \frac{1}{(2 \pi)^{3} \tau_{0}} \int \mathrm{d}^{2} \mathrm{p}_{\mathrm{T}} \mathrm{d} \mu \omega\left(\tau_{0}\right) n_{\mathrm{B}}\left(\tau_{0}\right) \\
& -\frac{1}{2(2 \pi)^{3} \tau_{0}} \int \mathrm{d}^{2} \mathrm{p}_{\mathrm{T}} \mathrm{d} \mu \omega\left(\tau_{0}\right)\left[K\left(\tau_{0}\right)-1\right]
\end{aligned}
$$

While the first term is finite, the second is not due to the behavior of the $K$ function for large values of its effective argument, which is $m_{T} \tau_{0}$, at fixed $\mu$ [see Eqs. (30) and (24)]. The asymptotic behavior for large transverse mass $m_{\mathrm{T}}$ of the $K$ function is derived in Appendix $\mathrm{C}$ and one has, at leading order,

$K\left(\tau_{0}\right)-1=\cosh 2 \Theta\left(\tau_{0}\right)-1 \simeq 2 \Theta^{2}\left(\tau_{0}\right) \simeq \frac{1}{8 m_{\mathrm{T}}^{2} \tau_{0}^{2}}$,

which makes the rightmost integral in Eq. (61) divergent. 
In conclusion, in order to have a finite stress-energy tensor, we are left with one option: to subtract the VEV's with respect to $\left|0_{\tau_{0}}\right\rangle$, which can be readily done by taking the limit $T\left(\tau_{0}\right) \rightarrow 0$ in Eq. (60) and subtracting what is left, taking into account that $\lim _{T \rightarrow 0} n_{B}=0$. We thus have

$$
\begin{aligned}
\Gamma_{\gamma}(\tau)_{\text {ren }}= & \frac{1}{(2 \pi)^{3} \tau} \int \mathrm{d}^{2} \mathrm{p}_{\mathrm{T}} \mathrm{d} \mu \omega(\tau)\left\{K_{\gamma}(\tau) K\left(\tau_{0}\right)\right. \\
& \left.-\operatorname{Re}\left[\Lambda_{\gamma}(\tau) \Lambda^{*}\left(\tau_{0}\right)\right]\right\} n_{\mathrm{B}}\left(\tau_{0}\right)
\end{aligned}
$$

which incorporates the relation between the energy density and the pressures.

It is interesting to study the behavior of the functions (63) at late times $\tau$, which means for large values of $m \tau$ (see Appendix C). In this limit, we have $\Theta(\tau) \rightarrow 0$, hence $K(\tau) \rightarrow 1$ and $\Lambda(\tau) \rightarrow 0$, implying that the Minkowskian vacuum is recovered asymptotically. This is also clear from Eq. (44), which shows that $\left|0_{\tau}\right\rangle \rightarrow\left|0_{M}\right\rangle$. For the energy density, at late times we have

$$
\begin{aligned}
\mathcal{E}(\tau)_{\text {ren }} \underset{\tau \rightarrow \infty}{\simeq} \frac{1}{(2 \pi)^{3} \tau} \int \mathrm{d}^{2} \mathrm{p}_{\mathrm{T}} \mathrm{d} \mu \omega(\tau) K\left(\tau_{0}\right) n_{\mathrm{B}}\left(\tau_{0}\right) \\
=\frac{1}{(2 \pi)^{3} \tau} \int \mathrm{d}^{2} \mathrm{p}_{\mathrm{T}} \mathrm{d} \mu \omega(\tau) n_{\mathrm{B}}\left(\tau_{0}\right)+\frac{1}{(2 \pi)^{3} \tau} \int \mathrm{d}^{2} \mathrm{p}_{\mathrm{T}} \mathrm{d} \mu \omega(\tau)\left[K\left(\tau_{0}\right)-1\right] n_{\mathrm{B}}\left(\tau_{0}\right) \\
=\frac{1}{(2 \pi)^{3} \tau} \int \mathrm{d}^{2} \mathrm{p}_{\mathrm{T}} \mathrm{d} \mu \omega(\tau) n_{\mathrm{B}}\left(\tau_{0}\right)+\frac{2}{(2 \pi)^{3} \tau} \int \mathrm{d}^{2} \mathrm{p}_{\mathrm{T}} \mathrm{d} \mu \omega(\tau) \sinh ^{2} \Theta\left(\tau_{0}\right) n_{\mathrm{B}}\left(\tau_{0}\right) .
\end{aligned}
$$

It can be shown that the first term in Eq. (64) is the classical free-streaming solution in Milne coordinates (see Appendix A), while the second term is a pure quantum-field correction due to the difference between vacua, since it vanishes only if $\Theta\left(\tau_{0}\right)=0$. Somewhat surprisingly, the quantum correction to energy density does not vanish at late times, and it can even be comparable with the classical term if the main argument of $\Theta\left(\tau_{0}\right)$, that is $m_{T} \tau_{0}$ is $\mathcal{O}(1)$, that is for an early decoupling of the system.

Similar expressions can be obtained for the pressures. For large times, the leading term of the $\Lambda_{\gamma}(\tau)$ function has an oscillating behavior $\sim \exp \left(-2 i m_{T} \tau\right)$ so the integrals in $\mathrm{p}_{\mathrm{T}}$ or $m_{\mathrm{T}}$ involving $\Lambda_{\gamma}(\tau)$ are expected to vanish as $\tau \rightarrow \infty$ (see Appendix C). Therefore, only the first term of Eq. (63) is left and one has

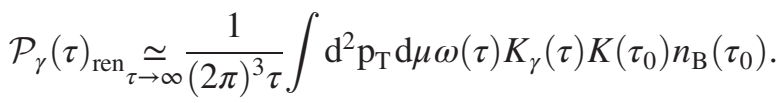

Also, at late times [see Appendix C, Eq. (C3)],

$$
\omega(\tau) K_{\gamma}(\tau) \underset{\tau \rightarrow \infty}{\simeq} \frac{m_{\mathrm{T}}^{2}+\gamma}{2 m_{\mathrm{T}}},
$$

so Eq. (65) becomes

$$
\begin{aligned}
\mathcal{P}_{\gamma}(\tau)_{\text {ren }} \underset{\tau \rightarrow \infty}{\simeq} & \frac{1}{(2 \pi)^{3} \tau} \int \mathrm{d}^{2} \mathrm{p}_{\mathrm{T}} \mathrm{d} \mu \frac{m_{\mathrm{T}}^{2}+\gamma}{2 m_{\mathrm{T}}} K\left(\tau_{0}\right) n_{\mathrm{B}}\left(\tau_{0}\right) \\
= & \frac{1}{(2 \pi)^{3} \tau} \int \mathrm{d}^{2} \mathrm{p}_{\mathrm{T}} \mathrm{d} \mu \frac{m_{\mathrm{T}}^{2}+\gamma}{2 m_{\mathrm{T}}} n_{\mathrm{B}}\left(\tau_{0}\right) \\
& +\frac{2}{(2 \pi)^{3} \tau} \int \mathrm{d}^{2} \mathrm{p}_{\mathrm{T}} \mathrm{d} \mu \frac{m_{\mathrm{T}}^{2}+\gamma}{2 m_{\mathrm{T}}} \sinh ^{2} \Theta\left(\tau_{0}\right) n_{\mathrm{B}}\left(\tau_{0}\right),
\end{aligned}
$$

with $\gamma$ from Eq. (54). Again, the first term on the right-hand side is the leading approximation of the classical freestreaming solution in Milne coordinates for large $m_{T} \tau$ and fixed $\mu$, whereas the second term is a pure quantum correction (see Appendix A).

As has been mentioned, the relative size of the quantum corrections with respect to the classical free streaming term can be non-negligible. As it appears from Eqs. (64) and (66), the relative size of the quantum corrections to the energy density and pressures is determined by the size of $\Theta\left(\tau_{0}\right)$. In general, the earlier the decoupling the larger is $\sinh ^{2} \Theta$ and the weight of the quantum correction. For a sufficiently large value of the dimensionless variable $m_{T} \tau_{0}$, the $\Theta\left(\tau_{0}\right)$ angle is given by the approximate formula in Eq. (62):

$$
\Theta\left(\tau_{0}\right) \simeq \frac{1}{4 m_{T} \tau_{0}}
$$

By using this approximation, it is possible to compute the relative corrections to the energy density and pressure as a function of the two independent dimensionless variables $m \tau_{0}$ and $m / T\left(\tau_{0}\right)$. For $m \tau_{0}=1.41$ the curves for the energy density and transverse as well as longitudinal pressures as a function of $m / T_{0}$ are shown in Fig. 2 . One observes that the relative corrections can be as large as several percent. The importance of these corrections decreases quickly as $m \tau_{0}$ increases, since the quantum corrections are inversely proportional to $m^{2} \tau_{0}^{2}$ in this limit. This means, however, that the small $m / T_{0}$ part of Fig. 2 must be interpreted as the limit of large initial temperatures, and not the limit of vanishing mass. One cannot use the approximation (62) in the massless limit, since the integrals diverge in the neighborhood of $p_{T}=0$, irrespective of the 


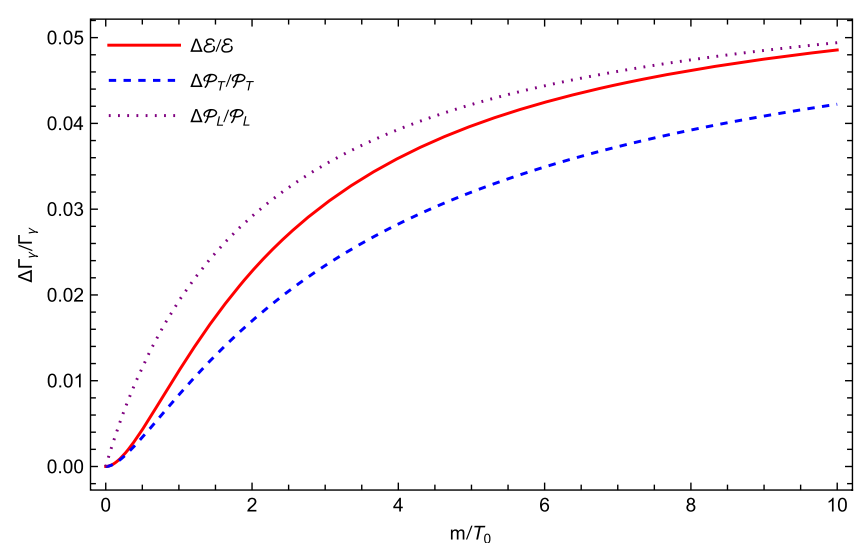

FIG. 2. Relative magnitude of the quantum corrections to the classical free-streaming solutions of energy density, transverse and longitudinal pressures, according to Eqs. (64) and (66), as a function of $m / T_{0}$ for $m \tau_{0}=1.41$.

value of $\tau_{0}$. It is possible, however, to directly compute the $m \rightarrow 0$ limit of Eq. (65) for small values of the remaining dimensionless parameter $T_{0} \tau_{0}$. The relative magnitude of the quantum corrections is of the order of $\sim 40 \%$ for $T_{0} \tau_{0} \simeq 0.1$, and they become the dominant contribution when $T_{0} \tau_{0}$ decreases. Therefore, their phenomenological relevance in expanding systems depends on the interplay between the mass of the particle, the dynamical decoupling time, and the initial temperature (hence energy density), which in turn depends on the dynamical preequilibrium stage [25-27].

\section{ENTROPY CURRENT}

The need of subtracting the vacuum $\left|0_{\tau_{0}}\right\rangle$ to obtain a finite value for the stress-energy tensor for the free field has some interesting connection to the way the entropy and the entropy current of a relativistic fluid in local thermodynamic equilibrium are calculated. This problem has been approached in the framework of the relativistic density operator in Ref. [28]. We first observe that the entropy of a relativistic fluid in local equilibrium,

$$
S=-\operatorname{tr}\left(\widehat{\rho}_{\mathrm{LE}} \log \widehat{\rho}_{\mathrm{LE}}\right),
$$

with $\widehat{\rho}_{\text {LE }}$ given by Eq. (49), is independent of the vacuum subtraction because, as remarked in Sec. II, the density operator (49) turns out to be independent of any nonoperator term which is subtracted from the stress-energy tensor operator, as it cancels out in the ratio with the normalizing $Z_{\mathrm{LE}}$.

However, it was pointed out in Ref. [28] that, provided that the vacuum is nondegenerate, there is only one good choice of the vacuum if one has to make $\log Z_{\mathrm{LE}}$ extensive, i.e.,

$$
\log Z_{\mathrm{LE}}=\int_{\Sigma} \mathrm{d} \Sigma_{\mu} \phi^{\mu}
$$

and this is the vacuum (meant as the eigenvector with minimal eigenvalue) of the operator $\widehat{\Pi}(\tau)$, which we have denoted with $\left|0_{\tau}\right\rangle$. Therefore, ${ }^{2}$ the entropy current reads

$$
s^{\mu}=\phi^{\mu}+\left[\operatorname{Tr}\left(\widehat{\rho}_{\mathrm{LE}} \widehat{T}^{\mu \nu}\right)-\left\langle 0_{\tau}\left|\widehat{T}^{\mu \nu}\right| 0_{\tau}\right\rangle\right] \beta_{\nu},
$$

with

$$
\phi^{\mu}=\int_{1}^{\infty} \mathrm{d} \lambda\left\{\operatorname{Tr}\left[\widehat{\rho}_{\mathrm{LE}}(\lambda) \widehat{T}^{\mu \nu}\right]-\left\langle 0_{\tau}\left|\widehat{T}^{\mu \nu}\right| 0_{\tau}\right\rangle\right\},
$$

where $\widehat{\rho}_{\mathrm{LE}}(\lambda)$ is the operator defined by

$$
\widehat{\rho}_{\mathrm{LE}}(\lambda)=\frac{1}{Z_{\mathrm{LE}}(\lambda)} \exp \left(-\lambda \int_{\Sigma} \mathrm{d} \Sigma_{\mu} \widehat{T}^{\mu \nu} \beta_{\nu}\right) .
$$

The renormalized value

$$
T_{\mathrm{LE}}^{\mu \nu}=\operatorname{Tr}\left(\widehat{\rho}_{\mathrm{LE}} \widehat{T}^{\mu \nu}\right)-\left\langle 0_{\tau}\left|\widehat{T}^{\mu \nu}\right| 0_{\tau}\right\rangle
$$

of the stress-energy tensor in local thermodynamic equilibrium with subtraction of the VEV with respect to $\left|0_{\tau}\right\rangle$ can be found by taking the limit $T(\tau) \rightarrow 0$, as we have seen in Sec. III A. Hence, for the free scalar field, it is readily found from Eq. (56) that we are left with the classical expression:

$$
T^{\mu \nu}(x)_{\mathrm{LE}}=\int \frac{\mathrm{d}^{3} p}{\varepsilon} p^{\mu} p^{\nu} \frac{1}{\mathrm{e}^{\beta(x) \cdot p}-1} .
$$

It is now easy to show that $\phi^{\mu}=\mathcal{P}_{\mathrm{LE}} \beta^{\mu}$, with $\mathcal{P}_{\mathrm{LE}}$ being the pressure in Eq. (57), and that the entropy current coincides with the classical equilibrium expression,

$$
s^{\mu}=\left(\mathcal{E}_{\mathrm{LE}}+\mathcal{P}_{\mathrm{LE}}\right) \beta^{\mu},
$$

where $\mathcal{E}_{\mathrm{LE}}$ and $\mathcal{P}_{\mathrm{LE}}$ are related by the usual equation of state of a free relativistic gas, without apparent quantum correction.

We end this section by discussing the entropy-production rate equation established in Refs. [10,11] (for a derivation see Ref. [19]), which for $\zeta=0$ reads

$$
\nabla_{\mu} s^{\mu}=\left(T^{\mu \nu}-T_{\mathrm{LE}}^{\mu \nu}\right) \nabla_{\mu} \beta_{\nu} .
$$

In the above equation it is usually understood that $T^{\mu \nu}$ and $T_{\mathrm{LE}}^{\mu \nu}$ are the renormalized stress-energy tensor expectation values, fulfilling the constraint equation (4), and usually obtained by subtracting the Minkowski VEV of both.

\footnotetext{
${ }^{2}$ In this section, for the sake of simplicity, we assume vanishing chemical potentials, that is $\zeta=0$; the extension of these arguments to a nonvanishing chemical potential is straightforward.
} 
However, in our case, in order to obtain finite values for the constraint equation (4) and to find an appropriate expression of the entropy current, we need to subtract different VEV's, as we have seen. In particular,

$$
\begin{aligned}
T_{\mathrm{LE}}^{\mu \nu} & =\operatorname{Tr}\left(\widehat{\rho}_{\mathrm{LE}} \widehat{T}^{\mu \nu}\right)-\left\langle 0_{\tau}\left|\widehat{T}^{\mu \nu}\right| 0_{\tau}\right\rangle, \\
T^{\mu \nu} & =\operatorname{Tr}\left(\widehat{\rho} \widehat{T}^{\mu \nu}\right)-\left\langle 0_{\tau_{0}}\left|\widehat{T}^{\mu \nu}\right| 0_{\tau_{0}}\right\rangle .
\end{aligned}
$$

One may thus wonder whether such a difference in the VEV subtraction introduces a new quantum term in the entropy production rate. The answer is again no, provided that

(i) the renormalized expectation value $T^{\mu \nu}$ is finite;

(ii) the renormalized expectation value $T^{\mu \nu}$ fulfills the continuity equation;

(iii) the renormalized expectation value in local equilibrium $T_{\mathrm{LE}}^{\mu \nu}$ fulfills the constraint (4).

The proof of Eq. (68) [19] can be shown to hold.

\section{SUMMARY AND CONCLUSIONS}

To summarize, we have studied a relativistic quantum fluid with longitudinal boost invariance, which, for the free scalar field, is an exactly solvable nonequilibrium problem, further developing and extending the results of Refs. $[13,14]$. By using the nonequilibrium density operator, we have derived an exact solution for the stress-energy tensor and the entropy current for the free scalar field initially in local thermodynamic equilibrium. The most remarkable feature of the solution is the difference between the vacuum of the density operator and the familiar vacuum of the field in Minkowski space-time. We have found that a finite, renormalized value of the stress-energy tensor can be achieved only by subtracting the vacuum of the density operator, and not the vacuum of the field; in practice, there is only one option. With respect to the known classical freestreaming solution, we have found quantum corrections related to the difference between the vacuum of the density operator and the Minkowski vacuum. It should also be emphasized that the quantum corrections do not depend on the system of coordinates used, the density operator being written in a fully covariant form. These corrections are numerically relevant for an early decoupling of the field, that is if $m \tau, T \tau$ are $\mathcal{O}(1)$ or smaller, where $\tau$ is the hyperbolic time; in this case they survive at late times and affect the relation between energy density and pressure as compared to the classical free-streaming case. These effects might be phenomenologically relevant whenever a quantum relativistic system expands and decouples. Their impact crucially depends on the interplay between the mass and the time of decoupling as well as the decoupling temperature.

\section{ACKNOWLEDGMENTS}

D. R. would like to express his gratitude to D. H. R. for his kind hospitality and support at the Institut für Theoretische Physik of Goethe University Frankfurt am Main during the course of this work. The work of L. T. and D. H. R. was supported by the Deutsche Forschungsgemeinschaft (DFG, German Research Foundation) through the CRC-TR 211 "Strong-interaction matter under extreme conditions,"Project No. 315477589 TRR 211.

\section{APPENDIX A: FREE STREAMING IN MILNE COORDINATES}

The collisionless Boltzmann equation in classical relativistic kinetic theory reads

$$
p \cdot \partial f(x, \mathbf{p})=0,
$$

and its explicit solution in Cartesian coordinates is

$$
f(x, \mathbf{p})=f_{0}\left(\mathbf{x}-\frac{t-t_{0}}{\varepsilon} \mathbf{p}, \mathbf{p}\right),
$$

where $f_{0}(\mathbf{x}, \mathbf{p})=f\left(t_{0}, \mathbf{x} ; \mathbf{p}\right)$ is the initial condition in a generic inertial reference frame, and $\varepsilon=\sqrt{m^{2}+p^{2}}$ is the (on-shell) energy.

In longitudinal boost-invariant symmetry, the initial condition is given at some Milne time $\tau_{0}$ rather than a time $t_{0}$ in Cartesian coordinates. Nevertheless, there is a very simple solution in this case, too. Since the distribution function is a scalar, it must be invariant under the symmetry transformations at stake, that are longitudinal boosts as well as rotations and translations in the transverse plane. Hence, it depends only on the independent scalars that may be formed with combinations of space-time and momentum vector which are invariant under the group of transformations $\operatorname{ISO}(2) \otimes \operatorname{SO}(1,1)$. These scalars are

$$
\tau=\sqrt{t^{2}-z^{2}}, \quad p_{\mathrm{T}}=\sqrt{p_{x}^{2}+p_{y}^{2}}, \quad w=z \varepsilon-t p^{z} .
$$

The last variable can be shown to be equivalent to the covariant component $p_{\eta}$ of the four-momentum vector in Milne coordinates. Indeed, there is a fourth invariant scalar:

$$
v=t \varepsilon-z p^{z}=\tau \sqrt{m^{2}+p_{\mathrm{T}}^{2}+\frac{w^{2}}{\tau^{2}}},
$$

but it is redundant because of the on-shell condition (and positivity) of the energy and because $t>|z|$ in the future light cone. The reflection invariance (see Sec. II) makes $f$ dependent on the square of $w$ rather than just $w$. By utilizing these arguments, Eq. (A1) becomes 


$$
\frac{v}{\tau} \frac{\partial}{\partial \tau} f\left(\tau, p_{\mathrm{T}}, w^{2}\right)=0,
$$

since the contribution in the partial derivatives with respect to $w$ cancels out. The free-streaming solution is then very simple, a constant in $\tau$ :

$$
f\left(\tau, p_{\mathrm{T}}, w^{2}\right)=f\left(\tau_{0}, p_{\mathrm{T}}, w\right) \equiv f_{0}\left(p_{\mathrm{T}}, w^{2}\right) .
$$

We are now in a position to calculate the free-streaming solution for the stress-energy tensor from its classical kinetic definition:

$$
\begin{aligned}
T^{\mu \nu}= & \frac{1}{(2 \pi)^{3}} \int \frac{\mathrm{d}^{3} \mathrm{p}}{\varepsilon} p^{\mu} p^{\nu} f \\
& \Rightarrow\left\{\begin{array}{l}
\mathcal{E}=u_{\mu} u_{\nu} T^{\mu \nu}=\frac{1}{(2 \pi)^{3}} \int \frac{\mathrm{d}^{3} \mathrm{p}}{\varepsilon} \frac{v^{2}}{\tau^{2}} f, \\
\mathcal{P}_{\mathrm{T}}=\frac{1}{2}\left(\widehat{i}_{\mu} \widehat{i}_{\nu}+\widehat{j}_{\mu} \widehat{j}_{\nu}\right) T^{\mu \nu}=\frac{1}{(2 \pi)^{3}} \int \frac{\mathrm{d}^{3} \mathrm{p}}{\varepsilon} \frac{p_{\mathrm{T}}^{2}}{2} f, \\
\mathcal{P}_{\mathrm{L}}=\widehat{\eta}_{\mu} \widehat{\eta}_{\nu} T^{\mu \nu}=\frac{1}{(2 \pi)^{3}} \int \frac{\mathrm{d}^{3} \mathrm{p}}{\varepsilon} \frac{w^{2}}{\tau^{2}} f,
\end{array}\right.
\end{aligned}
$$

and by changing the integration variables

$$
w=z \varepsilon-t p^{z} \Rightarrow \mathrm{d} w=\left|-\frac{v}{\varepsilon}\right| \mathrm{d} p^{z} \Rightarrow \frac{\mathrm{d} p^{z}}{\varepsilon}=\frac{\mathrm{d} w}{v},
$$

one obtains

$$
\begin{aligned}
\mathcal{E} & =\frac{1}{(2 \pi)^{3}} \int \mathrm{d}^{2} \mathrm{p}_{\mathrm{T}} \frac{\mathrm{d} w}{v} \frac{v^{2}}{\tau^{2}} f_{0}\left(p_{\mathrm{T}}, w^{2}\right) \\
& =\frac{1}{(2 \pi)^{3} \tau} \int \mathrm{d}^{2} \mathrm{p}_{\mathrm{T}} \mathrm{d} w \sqrt{m_{\mathrm{T}}^{2}+\frac{w^{2}}{\tau^{2}}} f_{0}\left(p_{\mathrm{T}}, w^{2}\right),
\end{aligned}
$$

and

$$
\begin{aligned}
& \mathcal{P}_{\mathrm{T}}=\frac{1}{(2 \pi)^{3}} \int \mathrm{d}^{2} \mathrm{p}_{\mathrm{T}} \frac{\mathrm{d} w}{v} \frac{p_{\mathrm{T}}^{2}}{2} f_{0}\left(p_{\mathrm{T}}, w^{2}\right)=\frac{1}{(2 \pi)^{3} \tau} \int \frac{\mathrm{d}^{2} \mathrm{p}_{\mathrm{T}} \mathrm{d} w}{\sqrt{m_{\mathrm{T}}^{2}+w^{2} / \tau^{2}}} \frac{p_{\mathrm{T}}^{2}}{2} f_{0}\left(p_{\mathrm{T}}, w^{2}\right), \\
& \mathcal{P}_{\mathrm{L}}=\frac{1}{(2 \pi)^{3}} \int \mathrm{d}^{2} \mathrm{p}_{\mathrm{T}} \frac{\mathrm{d} w}{v} \frac{w^{2}}{\tau^{2}} f_{0}\left(p_{\mathrm{T}}, w^{2}\right)=\frac{1}{(2 \pi)^{3} \tau} \int \frac{\mathrm{d}^{2} \mathrm{p}_{\mathrm{T}} \mathrm{d} w}{\sqrt{m_{\mathrm{T}}^{2}+w^{2} / \tau^{2}}} \frac{w^{2}}{\tau^{2}} f_{0}\left(p_{\mathrm{T}}, w^{2}\right) .
\end{aligned}
$$

The change of variable introduces an explicit dependence on the proper time in the integral.

Equations (A8) and (A9) are the classical relativistic expressions for the energy density and pressures of a freestreaming gas and coincide with the leading terms obtained in Sec. V with the substitution $w \rightarrow \mu$ and with the initial distribution equal to the local equilibrium Bose-Einstein distribution function $f_{0}=n_{\mathrm{B}}^{0}$.

\section{APPENDIX B: MINKOWSKI VACUUM EXPECTATION VALUES}

In order to calculate the scalars $\Gamma_{\gamma}(\tau)_{M}$ of the stressenergy tensor in the Minkowski vacuum, we take advantage of it being annihilated by all the $\widehat{b}_{\mathrm{p}}$ 's as it is clear from Eq. (23). Hence, the only product of $\widehat{b}_{\mathrm{p}}$ and $\widehat{b}_{\mathrm{p}}^{\dagger}$ with nonvanishing expectation value with respect to $\left|0_{M}\right\rangle$ is $\widehat{b}_{\mathrm{p}} \widehat{b}_{\mathrm{p}^{\prime}}^{\dagger}$, and using the commutation relations (22),

$$
\begin{aligned}
\left\langle 0_{M}\left|\widehat{b}_{\mathrm{p}} \widehat{b}_{\mathrm{p}^{\prime}}^{\dagger}\right| 0_{M}\right\rangle & =\left\langle 0_{M}\left|\widehat{b}_{\mathbf{p}^{\prime}}^{\dagger} \widehat{b}_{\mathrm{p}}\right| 0_{M}\right\rangle+\left\langle 0_{M}\left|\left[\widehat{b}_{\mathrm{p}}, \widehat{b}_{\mathbf{p}^{\prime}}^{\dagger}\right]\right| 0_{M}\right\rangle \\
& =\delta^{2}\left(\mathbf{p}_{\mathrm{T}}-\mathbf{p}_{\mathrm{T}}^{\prime}\right) \delta\left(\mu-\mu^{\prime}\right) .
\end{aligned}
$$

We can now replace these VEV's to obtain $\Gamma_{\gamma}(\tau)_{M}$ in the stress-energy tensor expression contracted with suitable vectors. For instance, for the energy density, we can use Eq. (49) by simply replacing the local equilibrium expectation values with those in the Minkowski vacuum and obtain

$$
\begin{aligned}
\mathcal{E}(\tau)_{M} & \equiv\left\langle 0_{M}\left|\widehat{T}^{\mu \nu} u_{\mu} u_{\nu}\right| 0_{M}\right\rangle=\int \frac{\mathrm{d}^{2} \mathrm{p}_{\mathrm{T}} \mathrm{d} \mu}{4(4 \pi)^{2}}\left(\left|\partial_{\tau} h\right|^{2}+\omega^{2}|h|^{2}\right) \\
& =\int \frac{\mathrm{d}^{2} \mathrm{p}_{\mathrm{T}} \mathrm{d} \mu}{(2 \pi)^{3} \tau} \omega \frac{K}{2}
\end{aligned}
$$

Similarly, for the pressures, one finds

$$
\Gamma_{\gamma}(\tau)_{M}=\int \frac{\mathrm{d}^{2} p_{T} \mathrm{~d} \mu}{(2 \pi)^{3} \tau} \omega \frac{K_{\gamma}}{2} .
$$

\section{APPENDIX C: ASYMPTOTICS}

It is interesting to study the behavior of the stress-energy tensor and related quantities for late times $\tau$. With 


$$
h(\tau)=-i \mathrm{e}^{\frac{\pi}{2} \mu} \mathrm{H}_{i \mu}^{(2)}\left(m_{\mathrm{T}} \tau\right),
$$

one can make use of the asymptotic expansion for large arguments [23]

$\mathrm{H}_{\nu}^{(2)}(x) \sim \sqrt{\frac{2}{\pi x}} \mathrm{e}^{-i\left(x-\frac{\pi}{2} \nu-\frac{\pi}{4}\right)} \sum_{n} \frac{1}{(2 i x)^{n}} \frac{\Gamma(\nu+1 / 2+n)}{n ! \Gamma(\nu+1 / 2-n)}$,

which is valid for $\operatorname{Re}(\nu)>-1 / 2$ and $|\arg (x)|<\pi$. Making use of the property $z \Gamma(z)=\Gamma(z+1)$, substituting $x=m_{\mathrm{T}} \tau$ and $\nu=i \mu$, and plugging this into Eq. (C1) we get

$$
h(\tau) \sim \sqrt{\frac{-2 i}{\pi m_{\mathrm{T}} \tau}} \mathrm{e}^{-i m_{\mathrm{T}} \tau} \sum_{n} \frac{1}{\left(2 i m_{\mathrm{T}} \tau\right)^{n}} \frac{\left(i \mu+\frac{1}{2}-n\right)^{(2 n)}}{n !},
$$

valid for large $m_{\mathrm{T}} \tau$. Similarly, using the exact relation [23]

$$
z \partial_{z} \mathrm{H}_{\nu}^{(2)}(z)=\nu \mathrm{H}_{\nu}^{(2)}(z)-z \mathrm{H}_{\nu+1}^{(2)}(z)
$$

along with the expansion (C2), one obtains the expansion for the proper-time derivative $\partial_{\tau} h$ :

$$
\partial_{\tau} h(\tau) \sim-i m_{\mathrm{T}} \sqrt{\frac{-2 i}{\pi m_{\mathrm{T}} \tau}} \mathrm{e}^{-i m_{\mathrm{T}} \tau}\left[1+\sum_{n>0} \frac{1}{\left(2 i m_{\mathrm{T}} \tau\right)^{n}}\left(-2 i \mu \frac{\left(i \mu+\frac{3}{2}-n\right)^{(2 n-2)}}{(n-1) !}+\frac{\left(i \mu+\frac{3}{2}-n\right)^{(2 n)}}{n !}\right)\right] .
$$

In particular, retaining the terms up to first order (i.e., nextto-leading order) in $m_{\mathrm{T}} \tau$ we get

$$
\begin{aligned}
h(\tau) & \simeq \sqrt{\frac{-2 i}{\pi m_{\mathrm{T}} \tau}} e^{-i m_{\mathrm{T}} \tau}\left[1-\frac{i}{2 m_{\mathrm{T}} \tau}\left(i \mu-\frac{1}{2}\right)\left(i \mu+\frac{1}{2}\right)\right] \\
& =\sqrt{\frac{-2 i}{\pi m_{\mathrm{T}} \tau}} \mathrm{e}^{-i m_{\mathrm{T}} \tau}\left(1+i \frac{1+4 \mu^{2}}{8 m_{\mathrm{T}} \tau}\right), \\
\partial_{\tau} h(\tau) & \simeq-i m_{\mathrm{T}} \sqrt{\frac{-2 i}{\pi m_{\mathrm{T}} \tau}} \mathrm{e}^{-i m_{\mathrm{T}} \tau}\left(1-i \frac{3-4 \mu^{2}}{8 m_{\mathrm{T}} \tau}\right) .
\end{aligned}
$$

Feeding the above expansions into the definitions (52) and (53), we obtain

$$
\begin{gathered}
K_{\gamma} \simeq \frac{m_{\mathrm{T}}^{2}+\gamma}{2 m_{\mathrm{T}} \omega}, \\
\Lambda_{\gamma} \simeq \frac{1}{2 m_{\mathrm{T}} \omega} \mathrm{e}^{-2 i m_{\mathrm{T}} \tau}\left[-m_{\mathrm{T}}^{2}\left(1-i \frac{3-4 \mu^{2}}{4 m_{\mathrm{T}} \tau}\right)\right. \\
\left.+\gamma\left(1+i \frac{1+4 \mu^{2}}{4 m_{\mathrm{T}} \tau}\right)\right] .
\end{gathered}
$$

The rest is of the order of $1 /\left[m_{\mathrm{T}} \omega\left(m_{\mathrm{T}} \tau\right)^{2}\right]$ for $K_{\gamma}$ and $\exp \left(-2 i m_{\mathrm{T}} \tau\right) /\left[m_{\mathrm{T}} \omega\left(m_{\mathrm{T}} \tau\right)^{2}\right]$ for $\Lambda_{\gamma}$.

Equations (C3) and (C4) are very useful to study the large $p_{\mathrm{T}}$ (hence, large $m_{\mathrm{T}}$ ) behavior as well as the long-time behavior. For large $p_{\mathrm{T}}$, Eq. (C3) implies that $K \rightarrow 1$, hence to leading order $K-1$ is simply zero. However, from Eq. (C4) and the exact relation (34) one can obtain the terms up to second order. Indeed, for $\gamma=\omega^{2}$, in the large $m_{\mathrm{T}}$ limit,

$$
\Lambda \simeq \frac{1}{2 m_{\mathrm{T}} \tau} \mathrm{e}^{-2 i m_{\mathrm{T}} \tau},
$$

hence,

$$
|\Lambda|=\sinh 2 \Theta \simeq 2 \Theta=\frac{1}{2 m_{\mathrm{T}} \tau},
$$

and in the limit of large $m_{\mathrm{T}}$

$$
K-1=\cosh 2 \Theta-1 \simeq \frac{(2 \Theta)^{2}}{2} \simeq \frac{1}{8 m_{\mathrm{T}}^{2} \tau^{2}} .
$$

Similarly, the leading expressions at late time $\tau \rightarrow \infty$ can be derived. By using the asymptotic expansions (C3) and (C4) and expanding $\omega(\tau)$ and $\gamma(\tau)$ for large $\tau$ one obtains

$$
\begin{aligned}
& K_{\gamma} \simeq \frac{m_{\mathrm{T}}^{2}+\tilde{\gamma}}{2 m_{\mathrm{T}}^{2}}, \\
& \Lambda_{\gamma} \simeq \frac{1}{2 m_{\mathrm{T}}^{2}} \mathrm{e}^{-2 i m_{\mathrm{T}} \tau}\left[\tilde{\gamma}-m_{\mathrm{T}}^{2}+i \frac{m_{\mathrm{T}}^{2}\left(3-4 \mu^{2}\right)+\tilde{\gamma}\left(1+4 \mu^{2}\right)}{4 m_{\mathrm{T}} \tau}\right],
\end{aligned}
$$

at first order in $1 / \tau$, with $\tilde{\gamma}$ :

$$
\tilde{\gamma}=\lim _{\tau \rightarrow \infty} \gamma= \begin{cases}m_{\mathrm{T}}^{2}, & \text { for } \mathcal{E}, \\ -m^{2}, & \text { for } \mathcal{P}_{\mathrm{T}}, \\ -m_{\mathrm{T}}^{2}, & \text { for } \mathcal{P}_{\mathrm{L}} .\end{cases}
$$

Differently from $K_{\gamma}$, the $\Lambda_{\gamma}$ function is highly oscillatory in the $\tau \rightarrow \infty$ limit. In order to prove that its contribution to kinematic integrals such as in Eqs. (64), (66) vanishes at the leading order of the asymptotic expansion, it is necessary remember the limit, valid in the distribution sense

$$
\int \mathrm{d} x f(x)=1 \Rightarrow \lim _{\varepsilon \rightarrow 0} \frac{1}{\varepsilon} f(x / \varepsilon)=\delta(x) .
$$


The real and imaginary part of the phase in $(\mathrm{C} 8)$ read

$$
\cos \left(2 m_{T} \tau\right), \quad \sin \left(2 m_{T} \tau\right)
$$

since

$$
\begin{aligned}
& \int_{-\infty}^{\infty} \mathrm{d} x \frac{\sin (x)}{x}=\pi \Rightarrow \lim _{\varepsilon \rightarrow 0}\left(\frac{1}{\pi} \frac{\sin (x / \varepsilon)}{x}\right)=\delta(x), \quad(\mathrm{C} 12) \\
& \int_{-\infty}^{\infty} \mathrm{d} x \cos \left(x^{2}\right)=\sqrt{\pi} \Rightarrow \lim _{\varepsilon \rightarrow 0}\left(\frac{1}{\varepsilon \sqrt{\pi}} \cos \left[\left(\frac{x}{\varepsilon}\right)^{2}\right]\right)=\delta(x),
\end{aligned}
$$

and one can rewrite both the real and imaginary part of the phase as a delta family

$$
\lim _{\tau \rightarrow \infty} \sin \left(2 m_{T} \tau\right)=2 \pi m_{T} \delta\left(2 m_{T}\right)
$$

$\lim _{\tau \rightarrow \infty} \cos \left(2 m_{T} \tau\right)=\lim _{\tau \rightarrow \infty} \sqrt{\frac{\pi}{\tau}} \delta\left(\sqrt{2 m_{T}}\right)=\lim _{\tau \rightarrow \infty} 2 \sqrt{\frac{\pi m_{T}}{\tau}} \delta\left(2 m_{T}\right)$,

In both cases, for massive fields, the Dirac delta argument $m_{T}=0$ is outside the physical range and any integration in $\mathrm{p}_{T}$ yields zero.
[1] F. Becattini, L. Bucciantini, E. Grossi, and L. Tinti, Eur. Phys. J. C 75, 191 (2015).

[2] T. Hayata, Y. Hidaka, T. Noumi, and M. Hongo, Phys. Rev. D 92, 065008 (2015).

[3] M. Garbiso and M. Kaminski, J. High Energy Phys. 12 (2020) 112.

[4] N. Weickgenannt, E. Speranza, X. 1. Sheng, Q. Wang, and D. H. Rischke, Phys. Rev. D 104, 016022 (2021).

[5] L. Tinti, arXiv:2003.09268.

[6] H. T. Elze, J. Phys. G 28, 2235 (2002).

[7] P. Ruggiero, P. Calabrese, B. Doyon, and J. Dubail, Phys. Rev. Lett. 124, 140603 (2020).

[8] W. Li and G. Wang, Annu. Rev. Nucl. Part. Sci. 70, 1 (2020) and references therein.

[9] F. Becattini and M. A. Lisa, Annu. Rev. Nucl. Part. Sci. 70, 395 (2020) and references therein.

[10] D. N. Zubarev, A. V. Prozorkevich, and S. A. Smolyanskii, Theor. Math. Phys. 40, 821 (1979).

[11] Ch. G. Van Weert, Ann. Phys. (N.Y.) 140, 133 (1982).

[12] F. Becattini and E. Grossi, Phys. Rev. D 92, 045037 (2015).

[13] S. V. Akkelin, Eur. Phys. J. A 55, 78 (2019).

[14] S. V. Akkelin, Phys. Rev. D 103, 116014 (2021).

[15] W. Florkowski, A. Kumar, R. Ryblewski, and R. Singh, Phys. Rev. C 99, 044910 (2019).

[16] V. Roy, S. Pu, L. Rezzolla, and D. Rischke, Phys. Lett. B 750, 45 (2015).
[17] S. Pu, V. Roy, L. Rezzolla, and D. H. Rischke, Phys. Rev. D 93, 074022 (2016).

[18] I. Siddique, R. j. Wang, S. Pu, and Q. Wang, Phys. Rev. D 99, 114029 (2019).

[19] F. Becattini, M. Buzzegoli, and E. Grossi, Particles 2, 197 (2019).

[20] T. Padmanabhan, Phys. Rev. Lett. 64, 2471 (1990).

[21] R. C. Arcuri, N. F. Svaiter, and B. F. Svaiter, Mod. Phys. Lett. A 09, 19 (1994).

[22] V. Mukhanov and S. Winitzki, Introduction to Quantum Effects in Gravity (Cambridge University Press, Cambridge, England, 2007).

[23] I. S. Gradshteyn, I. M. Ryzhik, D. Zwillinger, and V. Moll, Table of Integrals, Series, and Products, 8th ed. (Academic Press, New York, 2014).

[24] L.C.B. Crispino, A. Higuchi, and G. E. A. Matsas, Rev. Mod. Phys. 80, 787 (2008).

[25] G. Nijs, W. van der Schee, U. Gürsoy, and R. Snellings, Phys. Rev. Lett. 126, 202301 (2021).

[26] G. Nijs, W. van der Schee, U. Gürsoy, and R. Snellings, Phys. Rev. C 103, 054909 (2021).

[27] J. Jankowski, S. Kamata, M. Martinez, and M. Spaliński, Phys. Rev. D 104, 074012 (2021).

[28] F. Becattini and D. Rindori, Phys. Rev. D 99, 125011 (2019). 